\title{
Feature analysis and the role of similarity in preattentive vision
}

\author{
HANS-CHRISTOPH NOTHDURFT \\ Max Planck Institute for Biophysical Chemistry, Göttingen, Germany
}

\begin{abstract}
Texture arrays of line elements at various orientations were used to study three phenomena of preattentive vision. Subjects were asked (1) to discriminate texture areas and to distinguish their form (experiments on texture segmentation); (2) to detect salient or vertical line elements (experiments on pop-out); and (3) to identify configurations of similar or dissimilar targets (experiments on grouping). Within the patterns, line orientation was systematically varied to distinguish the effect of differences between areas from the effect of similarity within areas. In all of the experiments, performance was found to depend on local orientation contrast at texture borders rather than on the analysis of line orientation itself. Texture areas were correctly identified only when the orientation contrast at the border well exceeded the overall variation of line orientation in the pattern. Similarly, only target elements with high local orientation contrast were detected fast and "in parallel." Targets with an orientation contrast lower than background variation required serial search. Preattentive grouping was found to depend on saliency, as defined by local orientation contrast, but not on the similarity of line elements. In addition to local orientation contrast, which played an important role in all of the visual phenomena studied, influences from the alignment of line elements with the outline of a figure were also seen.
\end{abstract}

Three spontaneous perceptual phenomena have been described as occurring in the visual inspection of line arrays with differently oriented elements: (1) The pattern may segregate into distinct areas, each of which appears to be separated from neighboring areas by a clearly visible (but physically nonexistent) texture border (texture segmentation; Beck, 1966b, 1972, 1982; Julesz, 1975, 1984; Olson \& Attneave, 1970). (2) Individual line elements at an orientation different from that of the rest of the pattern appear to be particularly salient and are instantaneously detected in search experiments (pop-out; Treisman, 1985, 1986; Treisman \& Gormican, 1988). (3) Lines at similar orientations may be seen as grouping together, forming distinct ensembles of elements within the line pattern (grouping; Beck, 1966a, 1967). Although these phenomena have been discovered independently of each other, and although they have been quantitatively assessed in different ways, they share important properties. They can all be achieved by preattentive visionthat is, without detailed analysis of the pattern-and they all seem to recruit from the same set of visual features. In general, elements that do not segregate in texture segmentation tasks also do not pop out from each other or provide perceptual grouping. Controversial reports, ac-

I would like to thank Peter Ruhlender for a suggestion concerning Experiment 5 and Jacob Beck, Rick Gurnsey, Lester Krueger, Jeremy Wolfe, and an anonymous referee for helpful comments on an earlier version of the manuscript. I am grateful to all of the subjects for patient cooperation and to Cyrilla Maelicke for correcting the English text. Correspondence should be addressed to $\mathrm{C}$. Nothdurft, Department of Neurobiology, Max Planck Institute for Biophysical Chemistry, P.O. Box 2841, W-3400 Göttingen, Germany (e-mail: nothdurft@ mike.dnet.gwdg.de). cording to which some properties provide texture segmentation but not pop-out (e.g., "crossings" and "noncrossings"; see Julesz \& Bergen, 1983; Treisman \& Gormican, 1988), may be due to different arrangements of elements in these tasks and to the resulting differences in spatial frequency composition of the pattern. Several experiments (Bergen \& Adelson, 1988; Gurnsey \& Browse, 1987; Nothdurft, 1990, 1991a) have suggested that crossings and noncrossings do not segregate because of their differences in line intersection but because of associated differences in the spatial frequency domain (cf. Nothdurft, 1990).

In describing the perceptual phenomena of grouping and segregation, Wertheimer (1923) stressed the aspect of similarity (Gleichheit) of elements, a view that also influenced later concepts of texture segmentation. Texture areas were thought to appear homogeneous if elements shared certain properties, and they were thought to segregate if their elements displayed different properties. Even in his early studies, however, Beck (1966a, 1967) conjectured that grouping was based on the dissimilarity between, rather than the similarity within, groups of line elements. He found that the judged similarity of texture elements failed to predict the degree to which they formed distinct perceptual groups and supposed that dissimilarities were detected by "difference units" (Beck, 1982) operating over retinal areas of various sizes. Grouping effects were assumed to be stronger for differences between large areas than for differences between small areas (Beck, Prazdny, \& Rosenfeld, 1983). Later experiments indicated that texture segmentation and pop-out are also strongly influenced by local variations in the pattern (Julesz, 1986; Nothdurft, 1985b; Sagi \& Julesz, 1987). Texture areas with globally identical feature statistics were shown to segregate when 
local discontinuities were introduced into the pattern, and, conversely, pronounced texture differences were shown to fail to segregate when the variations were made continuous (Nothdurft, 1985b, 1990).

In the present study, the role played by local texture differences in segmentation, pop-out, and grouping phenomena was investigated in detail, and the different contributions of orientation contrast and the analysis of orientation per se were compared. Preliminary data from some of the experiments have been reported recently (Nothdurft, 1991c). Although the work presented in this paper concentrates on variation in line orientation, which is an important feature in preattentive vision, the approach has recently been extended to the use of other stimulus dimensions such as color and motion (Nothdurft, 1991b). The data suggest similar properties for these parameters, with some interesting exceptions for color.

The experiments reported here followed three directions. (1) The strength of local orientation differences required for perceiving texture segmentation and pop-out was assessed quantitatively and measured as a function of the overall variation of line orientation in the pattern. (2) The speed in detecting certain line orientations was measured as a function of target orientation contrast. (3) Perceptual grouping was assessed quantitatively, and the strength of grouping of similar and dissimilar elements was compared.

\section{GENERAL METHOD}

Experiments were performed on adult subjects with normal or corrected-to-normal visual acuity, who were paid for their participation. The subjects sat about $2 \mathrm{~m}$ away from a monitor screen (CONRAC 7211) on which the stimuli appeared. Before and during stimulus presentation, they were asked to fixate a small spot on the screen that remained visible during the experiment.

The stimuli were texture patterns of bright lines (about $20^{\prime}$ long by $5^{\prime}$ wide) that could appear at various orientations. The raster width of the line arrangements was usually $35^{\prime}$ of visual angle; in some experiments, denser or less dense texture patterns were also tested.

Four different paradigms were used. In the experiments on texture segmentation, the subjects had to distinguish texture areas and to report the orientation of a texture bar that appeared in the center of the screen and could be either vertical or horizontal. In the popout experiments, the subjects either had to detect a particularly salient line element (at eccentricities of $1^{\circ}-2^{\circ}$ ) and to indicate its position relative to the fixation point ("left" or "right"), or to report the presence or absence of a certain line in the pattern (visual search experiments). In the grouping experiments, the subjects were asked to detect ensembles of line elements and to indicate their spatial configuration-for example, whether they formed triangles or circles. Note that this paradigm is different from the one originally used in grouping experiments, in which the strength of grouping by similarity was estimated by measuring its effect on grouping by differences in luminance (Beck, 1966b, 1967). However, the visibility of element configurations as measured here was found to be a direct indication of the strength with which these elements formed a distinct group. The figures used (triangles, circles) were arbitrary and were selected because of their ease of being detected, distinguished, and reported by subjects.

The stimuli were generated by computer (LSI 23) and stored on a display buffer from which they were displayed for a given time through the use of a noninterlaced video technique (frame rate: $50 \mathrm{~Hz}$ ). Computation time for generating a new picture was typically 2-3 sec. In some experiments, the stimuli were masked by the immediately following presentation of another pattern with lines at random orientations. In the configuration used, spatial resolution of the picture buffer was $256 \times 256$ pixels, at two luminance levels (line elements, background). The presentation time of the stimulus patterns was typically $100 \mathrm{msec}$ ( 5 frame repetitions) for nonmasked stimuli and $160 \mathrm{msec}(8 \mathrm{frames})$ for stimuli followed by a masking pattern. The mask itself was shown for $500 \mathrm{msec}(25$ frames). Screen luminances were set to $2 \mathrm{~cd} / \mathrm{m}^{2}$ (background) and $20 \mathrm{~cd} / \mathrm{m}^{2}$ (texture elements), resulting in a $1 \log _{10}$ luminance difference between texture elements and background $(\Delta L / L=9)$.

In all tasks (except Experiment 4), responses were based on global impression rather than detailed analysis of the pattern. When a new experiment was started, an initial time period was used to familiarize the subjects with the paradigm and the required reactions. The subjects directly entered their answers into the computer by pressing specified buttons on the keybord. After a short delay, the computer started the calculation of a new pattern, which was then displayed. To keep the time for computing a new picture as short as possible, line elements at various orientations were not generated individually but were read from memory with a resolution of $5^{\circ}$ in line orientation.

\section{TEXTURE SEGMENTATION FROM LOCAL DISCONTINUITIES}

\section{Experiment 1: Segmentation of Nonuniform Texture Areas}

The aim of this experiment was to distinguish the effect of local dissimilarity at a texture border and that of global similarity within texture areas on perceived segmentation. Because these two aspects would be indistinguishable with the use of texture patterns in which all lines within an area had the same orientation (Figure 1A), measurements were extended to patterns with nonuniform texture areas in which line elements varied systematically in orientation (Figure 1B). Texture patterns were constructed from two parameters, the continuous variation of line orientation in the background of the pattern (the background orientation shift, $\Delta_{\text {bg }}$, indicating the difference in orientation between neighboring elements along rows and columns in the pattern) and an independently adjustable (usually larger) local change in line orientation at the outlines of the texture bar (texture border orientation contrast, $\Delta_{\mathrm{tb}}$ ). Both parameters were systematically varied, and the various conditions were intermixed and shown in random order, with actual line orientations chosen at random for each presentation. The data shown below represent an average of 60 or more stimulus presentations of each $\Delta_{\mathrm{tb}} / \Delta_{\mathrm{bg}}$ combination. The background orientation shift was usually positive (lines rotated clockwise from left to right and from top to bottom), but it could be inverted at random positions, causing the reversal of orientation shift. Such reversals in the flow field of the pattern were often applied when the variation of background orientations was large, in order to avoid dominant macrostructures at oblique orientations. The stimulus patterns were shown for $100 \mathrm{msec}$; they were not masked afterwards. The screen distance was $220 \mathrm{~cm}$. 


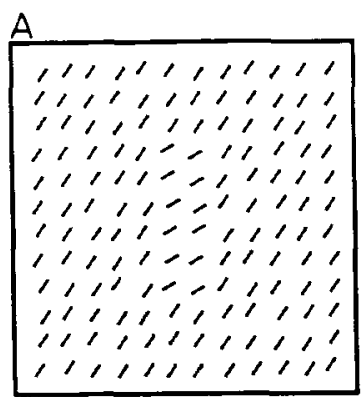

B

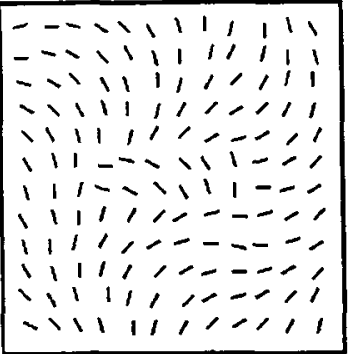

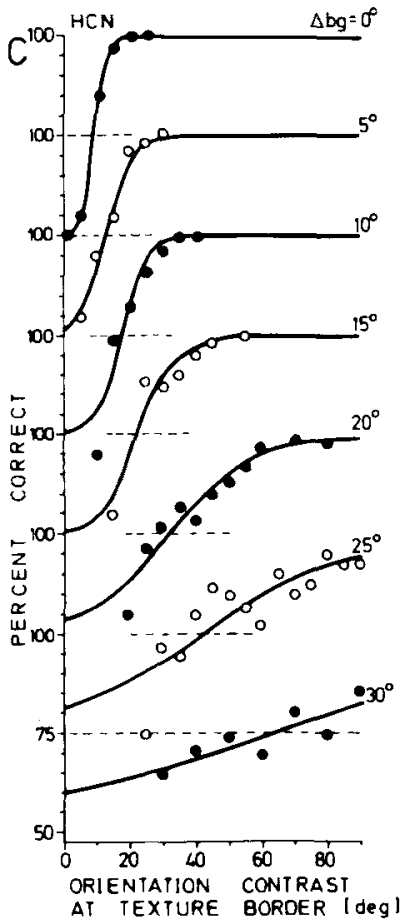

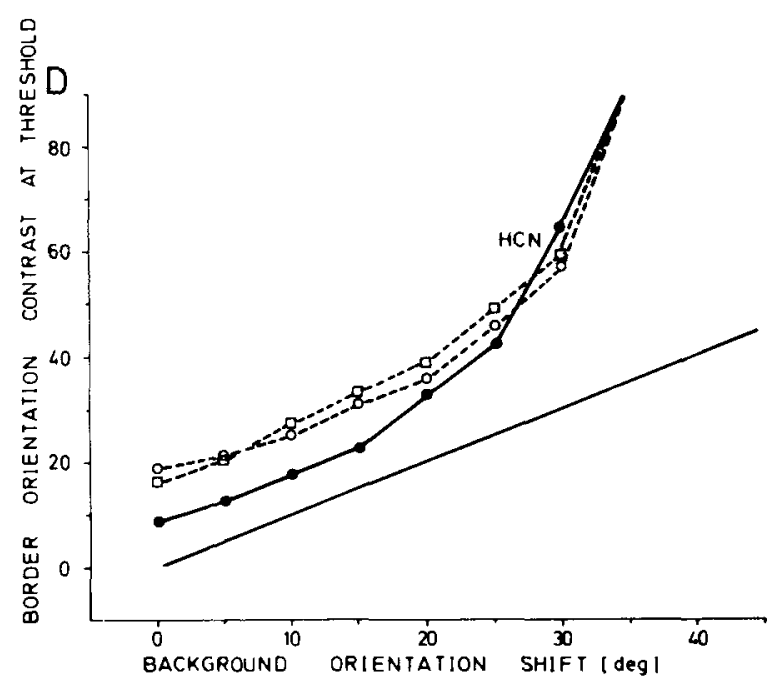

Figure 1. Segmentation of nonuniform texture areas. Panels $A$ and $B$ show stimulus patterns with systematic variation of two parameters, the overall orientation difference between neighboring line elements, $\Delta_{\mathrm{bz}}\left(0^{\circ}\right.$ in $\mathrm{A}, 15^{\circ}$ in $\left.\mathrm{B}\right)$, and the local shift in line orientation at the texture border, $\Delta_{t b}\left(30^{\circ}\right.$ in $\mathrm{A}, 90^{\circ}$ in B). Subjects had to detect the segregating texture area and to identify whether the global bar was vertical or horizontal. The actual orientation of line elements was randomly varied. Panel $\mathrm{C}$ gives correct responses for Subject H.C.N., sorted for similar background conditions (alternatingly filled and open circles) and fitted by psychometric curves. Different curves are shifted by $25 \%$ each and plotted together; dashed lines indicate $75 \%$ correct (threshold). In panel D, threshold orientation contrast $(75 \%$ correct from curves as in panel $\mathrm{C}$ ) is plotted against background orientation shift. Data from panel $\mathrm{C}$ are marked by filled circles; data from 2 other subjects are added (open circles). The straight line indicates the case $\Delta_{\mathrm{tb}}=\Delta_{\mathrm{b}_{\mathrm{g}}}$, which is seen as a continuous flow field with no segmentation.

Three subjects were tested in this experiment. For each of them, data were collected in two to three sessions, each of which lasted no longer than $2 \mathrm{~h}$. Correct responses for all border conditions on the same background variation were fitted by a psychometric function (Nothdurft, 1985a), whose $75 \%$ level was taken as a measure of threshold orientation contrast for texture segmentation on this background condition. Figure $1 \mathrm{C}$ shows the measurements for Subject H.C.N. Data points are sorted for the different background conditions. Intersections of fitted curves with the dashed lines (representing 75\% correct responses) are plotted in Figure 1D. Note that the threshold values of the border orientation contrast increase with background orientation shift. For uniform areas $\left(\Delta_{\mathrm{bg}}=0\right)$, local orientation contrast of less than $20^{\circ}$ (less than $10^{\circ}$ for Subject H.C.N.) was sufficient to provide texture segmentation, but even for an element-to-element variation of $20^{\circ}$, subjects could still correctly identify the global texture bar when orientation contrast at the texture border was made large enough. Up to values of about $15^{\circ}-20^{\circ}$, the required border contrast increased almost linearly with increasing background variation. Above $30^{\circ}$, subjects usually did not reach the $75 \%$ level for even maximal orientation contrast $\left(90^{\circ}\right)$ at the texture border. Note that upper limits of background variation for which segmentation could still be obtained were similar for all subjects, despite notable differences in threshold for small background variations.

These results cannot be explained by theories in which texture segmentation is based on element similarity. Only for zero background orientation shift could the analysis of line orientation per se predict the texture figures seen. For patterns with nonuniform texture areas, the analysis of element similarity would have predicted quite different segmentation figures. The figures seen were only to be expected if texture segmentation were linked to the detection of local dissimilarities larger than the overall variation within a pattern (Nothdurft, 1991c). Such a sensitivity to local dissimilarities (i.e., to orientation contrast) could be achieved from nonspecific summation of V1 cell responses, which have been found to increase with local orientation contrast (Knierim \& Van Essen, 1992; Van Essen et al., 1989).

An interesting observation in the course of these experiments was that the detection of texture bars did not depend on local orientation contrast alone, but was, to some extent, also influenced by the actual orientation of line elements near the texture border. Texture bars whose 
orientation lined up with the orientation of individual line elements were more easily detected than those whose line orientation and bar orientation differed considerably. This effect was only seen for patterns in which the orientation contrast at the texture border was so small that detection was near threshold. For both very small and very large contrast values, either no or all texture bars were detected, independently of the actual orientation of line elements. In the following series of experiments, these interactions between bar and line orientations were studied in more detail.

\section{Experiments 2A-2C: Alignment Effects in Texture Segmentation.}

The experimental paradigms and the stimuli in these experiments were similar to those used before, except that the orientation of line elements within the texture bar and the bar orientation itself (horizontal or vertical) were systematic parameters of the test. That is, line orientation was not random but was varied systematically in steps of $15^{\circ}$ (Experiments $2 \mathrm{~A}$ and $2 \mathrm{~B}$ ). Variations included cases in which line elements were aligned with the orientation of the texture bar (see Figure 2A), and others in which line orientation was orthogonal to it (see Figure 2C). Experiments $2 \mathrm{~A}$ and $2 \mathrm{~B}$ were restricted to a single background shift and border contrast condition $\left(\Delta_{\mathrm{bg}}=10^{\circ}\right.$; $\Delta_{\mathrm{tb}}=20^{\circ}$ ), which was selected because it produced detection rates near threshold in Experiment 1.

In Experiment $2 \mathrm{~A}, 5$ subjects were tested with this paradigm. Their mean responses are shown in Figures $3 \mathrm{~A}-3 \mathrm{C}$. The filled circles in Figure $3 \mathrm{~A}$ give the number of correct responses for patterns with a vertical texture bar; the open circles in Figure 3B give those for patterns with a horizontal bar. Because the background orientation shift was nonzero in all these patterns, only some of the lines within the texture bar exactly lined up with its orientation. The values on the abscissa in Figure $3 \mathrm{~A}\left(0^{\circ}\right.$ indicates vertical orientation, $90^{\circ}$ indicates horizontal orientation, and positive and negative values indicate righttilted and left-tilted lines, respectively) refer to the orientation of the most central line elements in the bar (cf.
Figure 2), the lines above right and below left from the center where the fixation point was located (not shown in Figure 2). Other line elements had slightly different orientations. However, for no element within the texture bar was the deviation from the given value larger than $30^{\circ}$. As is obvious from the distribution of filled and open circles, bars whose line elements were aligned with the bar's orientation were detected better than bars whose line elements were orthogonal to it.

When the data of both curves are pooled with respect to the relative orientation difference between line elements and the texture bar, the results (Figure $3 \mathrm{C}$ ) reveal a systematic dependence of correct bar detection on the alignment of border and element orientations.

Note that subjects performed worse than chance level for lines perpendicular to bar orientation, often identifying a vertical bar of horizontal lines as a horizontal bar, and vice versa. This indicates that subjects' decisions were biased by the orientation of texture elements in the center of the pattern. In order to see whether the bias was due to the configuration of central line elements alone or whether there was an additional effect from the alignment of texture elements with the texture border, the following two modifications of the experiment were made for control.

In Experiment 2B, the stimulus patterns were identical to those in Experiment 2A, but presentations also included patterns with no texture bar as blank trials. The background variation in these patterns was identical to that of the bar stimuli $\left(\Delta_{\mathrm{bg}}=10^{\circ}\right)$, and the central line elements were either horizontal or vertical. In addition to responding "vertical" or "horizontal," subjects could also reject a pattern by entering a "no bar" response. Nobar stimuli appeared in $30 \%$ of the presentations.

The curves in Figures 3D-3F represent the means of 3 subjects on 50 presentations of each bar condition. Their rate in seeing bars from no-bar stimuli (dashed lines) strongly depended on the orientation of the central line elements. In the context of the apparently weak visibility of the texture bars, the false alarm rate was well above zero. However, false alarms were strongly biased toward

\begin{tabular}{|c|c|c|c|}
\hline$\Delta$ & B & C & $\mathrm{D}$ \\
\hline 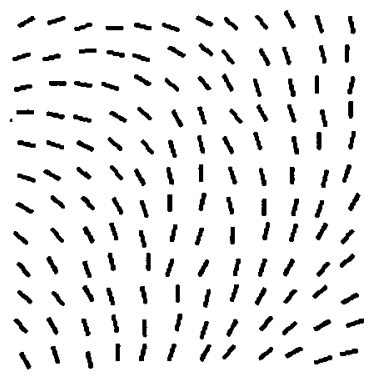 & 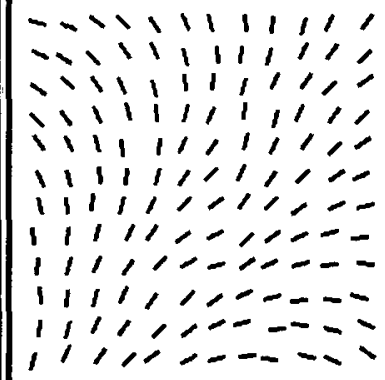 & 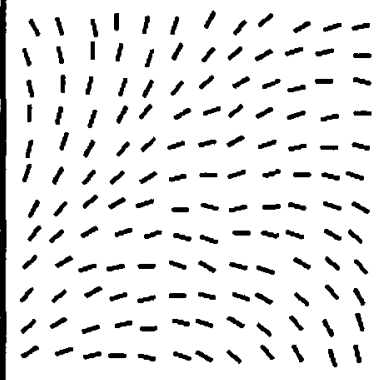 & 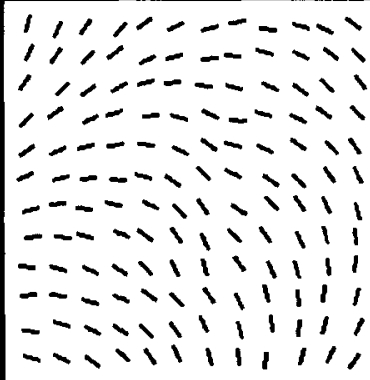 \\
\hline
\end{tabular}

Figure 2. Alignment effects in texture segmentation. Four patterns with constant stimulus conditions, according to Experiment 1 (vertical bar; $\Delta_{\mathrm{bs}}=10^{\circ} ; \Delta_{\mathrm{tb}}=20^{\circ}$ ), but different actual line orientations. Subjects detected the texture bar in panel $A$ more frequently than that in panel $\mathbf{C}$. 
A


$\mathrm{B}$

$$
\text { means of } 5 \text { subjects }
$$
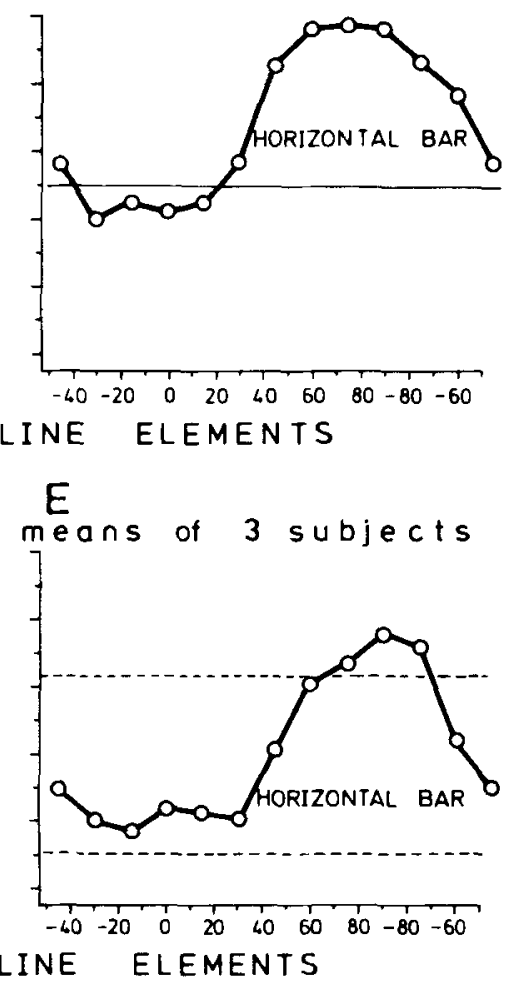

C

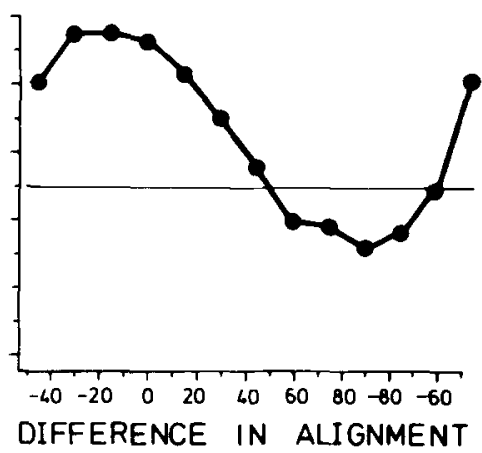

$\mathrm{F}$

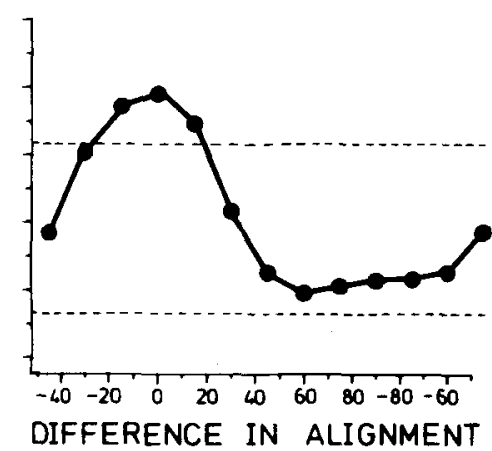

Figure 3. Alignment effects. Panels A-C give mean correct responses for 5 subjects' detection of vertical (A, filled symbols) and horizontal (B, open symbols) texture bars when orientation of line elements was systematically varied (Experiment $2 A$ ). Values on the abscissa refer to the orientation of central line elements $\left(0^{\circ}\right.$ indicates vertical orientation, and $90^{\circ}$ indicates horizontal orientation). Vertical bars are detected best when composed of vertical or near-vertical line elements $\left(A, 0^{\circ}\right)$, borizontal bars best when composed of horizontal or near-horizontal lines $\left(B, 90^{\circ}\right.$ ). Panel $C$ gives the averages of the curves in panels $A$ and $B$ with respect to alignment of texture bar and line elements. Panels D-F give the means for 3 subjects' detection of vertical and horizontal bars in the presence of blank trials (no-bar stimuli) with central lines at $0^{\circ}$ or $90^{\circ}$ (Experiment $2 B$ ). The plots are as in panels A-C, but subjects were asked to reject patterns without a texture bar. False alarms to blank stimuli are marked by dashed lines. Upper dashed lines indicate the rate of seeing aligned bars $\left(A\right.$, vertical bars from no-bar stimuli with central lines at $0^{\circ}$; $B$, horizontal bars from no-bar stimuli with central lines at $90^{\circ} ; \mathrm{C}$, mean). Lower dashed lines indicate the rate of seeing nonaligned bars (A, vertical bars from no-bar stimuli with central lines being horizontal $\left[90^{\circ}\right]$; B, horizontal bars from no-bar stimuli with vertical central lines $\left[0^{\circ}\right]$; $\mathrm{C}$, mean). Detection of aligned bars exceeds false alarm rate for aligned no-bar stimuli.

bars in an aligned orientation (upper dashed lines in Figures $3 \mathrm{D}$ and $3 \mathrm{E}$ ). Although the rates for seeing nonaligned bars were only slightly different between no-bar and bar stimuli (lower parts of the curves and lower dashed lines), there were consistent differences between the rates of seeing bars from aligned configurations (curve peaks and upper dashed lines). Subjects more often (correctly) detected the aligned texture bar than they (wrongly) guessed that such a bar was present in aligned no-bar textures. The net effect (hits minus false alarms) was $15 \%$ on the average from 100 bar presentations to each of the 3 subjects.

In order to see whether the size of this effect depended on orientation contrast at the texture border, we ran a final test on another modification. In this experiment (Experiment $2 \mathrm{C}$ ), variation of line orientation in the texture bar was restricted to the aligned and nonaligned cases for both vertical (see Figures 2A and 2C) and horizontal texture bars, and to vertical and horizontal no-bar stimuli. The advantage of this restriction was that the reduced number of test patterns allowed measurement of the subjects' performances with other border orientation contrasts as well. Tests were run in blocks of 80 bar and 40 no-bar presentations each, with constant texture border contrast within each block. Blocks with different border contrast were run in sequence, and all tests were repeated until data from 5-8 identical blocks (100-160 presentations per stimulus) had been accumulated. Background orientation shift was constant in all of these tests $\left(\Delta_{b z}=10^{\circ}\right)$. Examples of stimulus patterns are shown in Figure 4A; the different columns show patterns from different test blocks. In addition to the vertical and horizontal bars from vertical line elements plotted in Figure 4A (vertical referring to lines in the center), vertical and horizontal bars from horizontal line elements were also shown. The no-bar conditions (shown in the right-hand column of Figure 4A) were always included. 


\begin{tabular}{|c|c|c|c|}
\hline$\Delta_{t b}=60^{\circ}$ & $40^{\circ}$ & $30^{\circ}$ & no bar \\
\hline 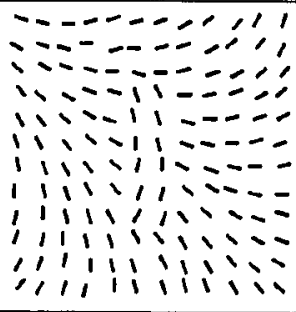 & 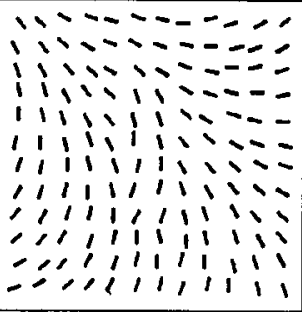 & 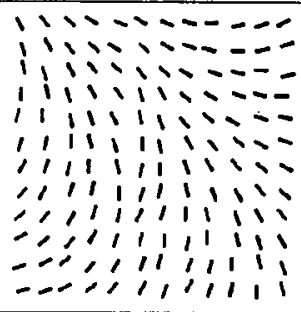 & 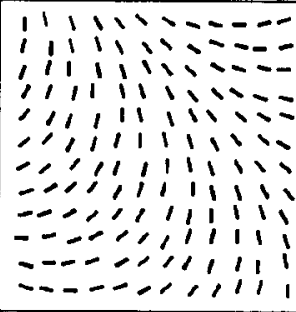 \\
\hline 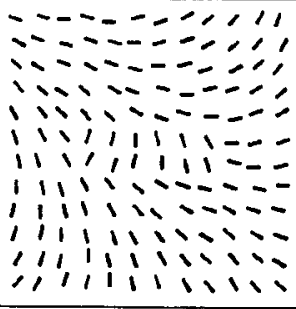 & 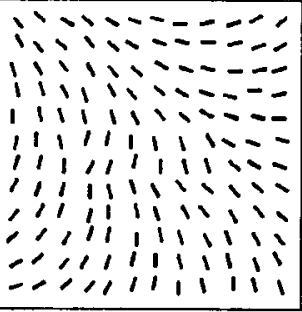 & 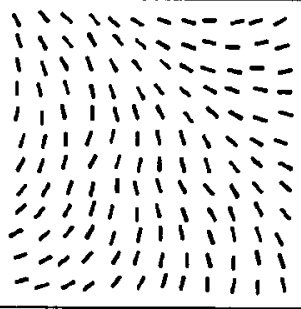 & 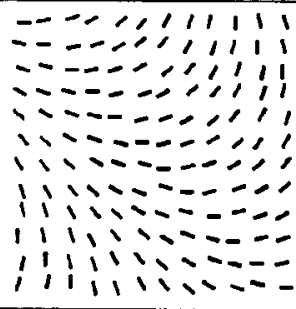 \\
\hline
\end{tabular}

B

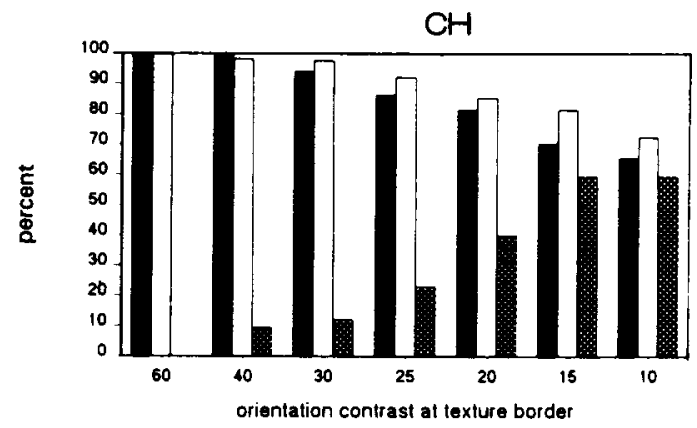

BARs seen

BJ

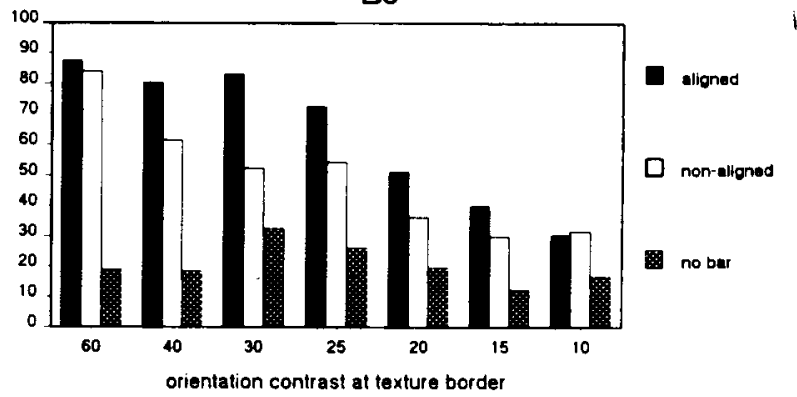

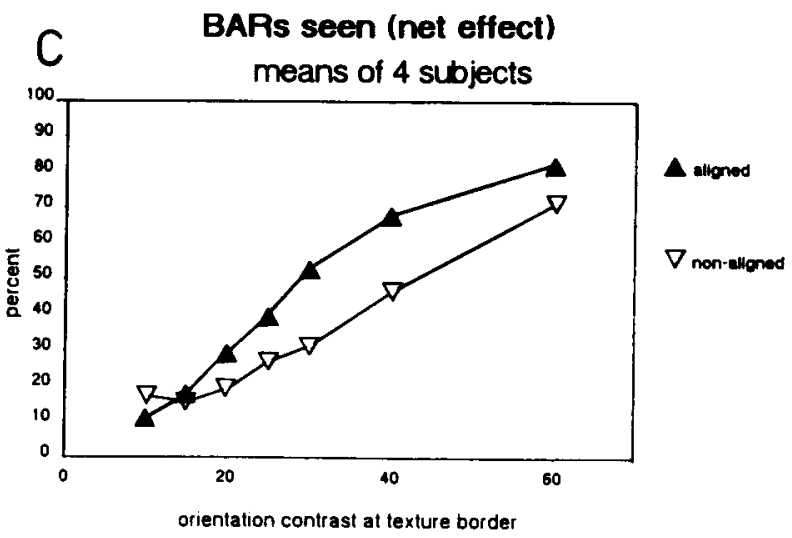

Figure 4. Alignment effects. Means are given for 4 subjects' detection of aligned and nonaligned bars at various texture border contrasts (Experiment 2C). The experimental condition is as in Figures 3D-F, but the tests were restricted to vertical and horizontal bars in aligned and nonaligned presentation, and to blank trials. A, examples of aligned vertical (upper row) and nonaligned horizontal bars (lower row); B, percentage of bars seen (by Subjects C.H. and B.J.), for different orientation contrasts at the texture border-histogram triplets indicate responses to aligned (filled), nonaligned (open), and no-bar (checkered) stimuli; $\mathbf{C}$, net effects (bar detection minus false alarms) for aligned (filled triangles) and nonaligned (open triangles) bars. 
Four subjects were tested in Experiment 2C; their mean data are plotted in Figure $4 \mathrm{C}$. Because the response characteristics differed between subjects, data from 2 of the subjects are separately shown in Figure 4B. Histograms plot the percentage of bars seen from aligned (filled), nonaligned (open), and no-bar (shaded) stimuli. For large orientation contrast at the texture border $\left(\Delta_{\mathrm{tb}}=60^{\circ}\right)$, detection rates for both aligned and nonaligned texture bars were high. With decreasing orientation contrast (and hence decreasing visibility of the bar), Subject C.H. responded with continuously increasing false alarm rates, whereas these rates remained fairly constant for Subject B.J. On the other hand, the detection rate of Subject B.J. strongly decreased with decreasing border contrast, whereas this decrease was smaller for Subject C.H. The resulting net effects were similar for both subjects. The means of net effects are shown, separately for aligned and nonaligned stimuli, in Figure 4C. With increasing orientation contrast at the texture border, the detection rates for both bar configurations increase, but the detectability of the aligned bars was always better than that of the nonaligned bars, in particular for intermediate texture border contrast (weak visibility of the texture bar). Thus, alignment of element orientation with that of the texture border helped in detection of the bar. Note that, although alignment effects were absent from the data of Subject C.H. (Figure 4B), the means (Figure 4C) show a clear effect even with her data included.

Usually, the majority of aligned bars was correctly identified (that is, the filled histograms in Figure 4B resemble merely correct responses), whereas subjects often took nonaligned bars for aligned bars in an incorrect orientation (that is, correct responses were fewer than indicated by the open histograms in Figure 4B). Subject B.J., for example, made almost no correct responses to nonaligned bars for texture border contrast below $25^{\circ}$. This bias of bar identification toward aligned versions was prominent for most subjects (in fact, for all except Subject C.H.) and is comparable to the bias found in Experiments $2 \mathrm{~A}$ and $2 \mathrm{~B}$.

Experiments 1 and 2 indicate that texture segmentation is primarily achieved from local dissimilarities in line orientation, with some additional contribution from alignment effects between line elements and the texture border. As discussed elsewhere (Nothdurft, 1991c), these observations cannot be explained by the notion that texture segmentation is based on the detection of certain features and the analysis of their similarity within texture areas. With increasing variation of line orientation within texture areas, local dissimilarities have to be enlarged in order to provide segmentation.

How the alignment of texture elements with bar orientation can account for the better visibility of the global figure on this basis remains an open question. Similar observations on the detection of aligned squares (Beck, Rosenfeld, \& Ivry, 1989) suggest that the ability of the visual system to detect line continuations may add to border extraction from local contrast. As Figure 4 illus- trates, alignment effects can affect texture segmentation to a different degree, for different subjects. Whereas performance was not affected at all, for Subject C.H., alignment effects were, in general, strong enough to make subjects see bars even in continuous and nonsegmented line arrays.

\section{REQUIREMENTS FOR VISUAL POP-OUT}

\section{Experiment 3: Pop-Out From Local Dissimilarity}

In this experiment, the role of orientation contrast in the pop-out phenomenon was studied. The stimuli were similar to those in Experiment 1, except that the targets were single elements instead of texture bars (Figure 5). The individual line elements (the targets) had a variable orientation contrast $\left(\Delta_{\mathrm{tg}}\right)$ larger than or equal to the element-to-element variation of line orientation in the background $\left(\Delta_{\mathrm{bg}}\right)$. The targets appeared at an eccentricity of $1^{\circ}-2^{\circ}$ to either side of the fixation point, and the subjects were asked to indicate whether they saw the target to the right (e.g., Figure 5A) or the left (e.g., Figure 5B) of the fixation point. The targets were defined by saliency, not by a certain orientation. They could, in fact, display any orientation during the course of a run. The task was immediately clear to all subjects, and the salient targets spontaneously caught their attention.

Stimulus presentation was identical to that in Experiment 1; the stimuli were displayed for $100 \mathrm{msec}$ and were not masked afterwards. Background orientation shift, $\Delta_{\text {bg }}$, and target orientation contrast, $\Delta_{\mathrm{tg}}$, were systematically varied, with random values for the actual line orientation of each pattern.

Figures 5A and 5B show examples of stimulus patterns with different background variations; in both patterns, the target is spontaneously detected. Correct responses for different target conditions on the same background variation were fitted by psychometric curves. The $75 \%$ levels of these curves were taken as the threshold orientation contrast for targets to pop out against this background. Threshold values of 5 subjects are plotted against background orientation contrast in Figures 5C and 5D.

Note that the operational definition of pop-out here is different from the usual reference to search time independent of display size. Considering the short presentation time (100 msec) and the large number of elements shown (121 and 144 items, respectively), the detection of the targets was instantaneous and probably independent of display size. This is also what subjects reported when doing the test. As will be shown in Experiment 4, the dependence of search time on the number of items displayed is closely related to the target's saliency, which, in fact, was measured here. Performance in detecting salient targets revealed curves similar to those for detecting texture bars (Figure 1C); that is, the detectability changed gradually with increasing target orientation contrast. In order to allow for comparisons between different background conditions, $75 \%$ correct responses were taken as an arbitrary reference. This, of course, does not imply 

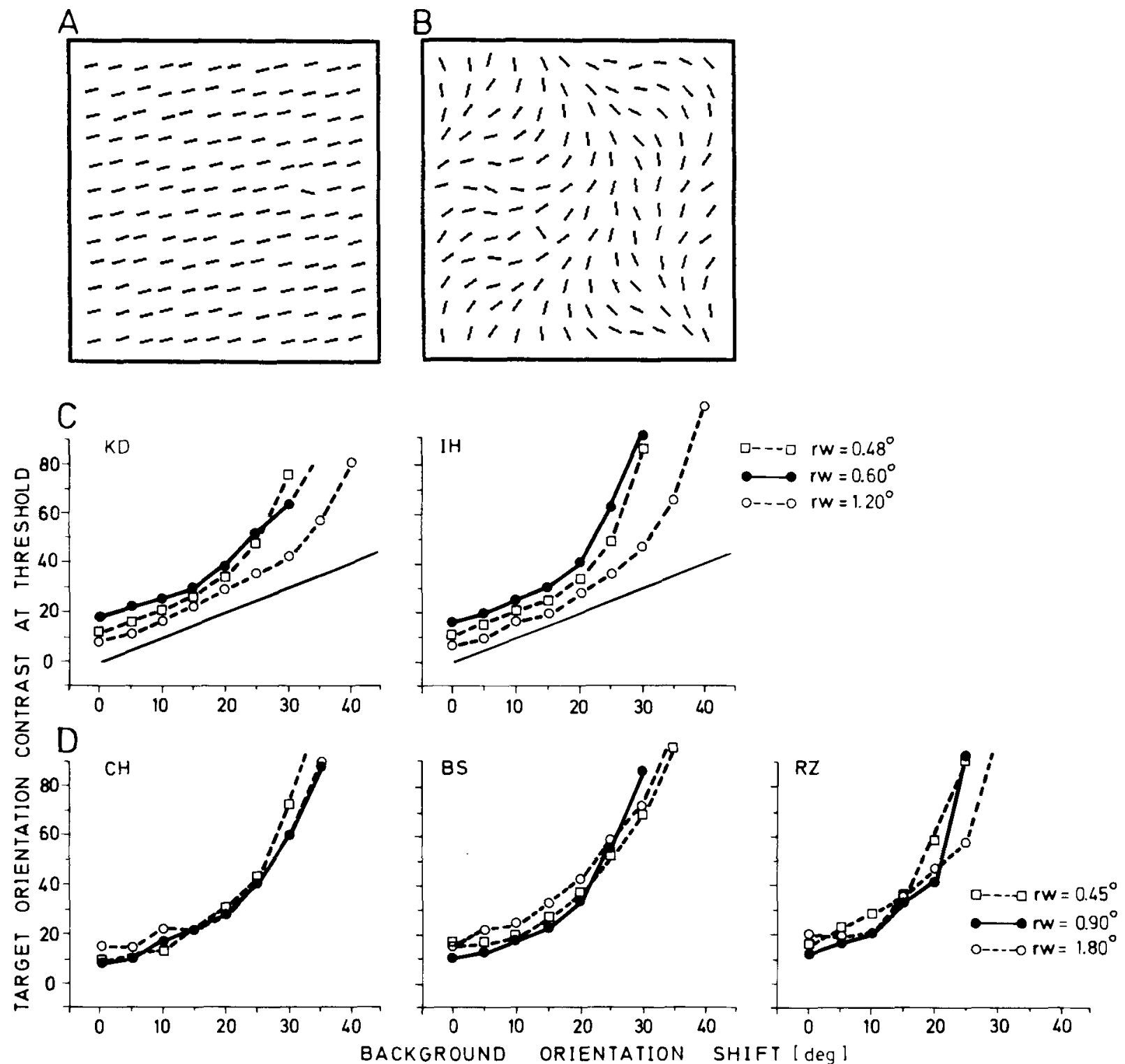

Figure 5. Pop-out from local dissimilarity. Subjects had to detect salient targets in an otherwise continuous line array and to indicate whether these appeared to the left or the right of the fixation point (in the middle of the pattern; not shown here). As in Experiment 1, variation of line orientation between elements (background orientation shift, $\Delta_{\mathrm{bl}}$ ) and local orientation contrast at target position ( $\Delta_{\mathrm{tg}}$ ) were the systematic parameters of the experiment (examples in $\mathrm{A}, \Delta_{\mathrm{bz}}=0^{\circ}, \Delta_{\mathrm{tz}}=30^{\circ}$, target on the right; $\mathrm{B}, \Delta_{\mathrm{bg}}=20^{\circ}, \Delta_{\mathrm{ig}}=90^{\circ}$, target on the left). Panels $C$ and $D$ show target orientation contrast at threshold, plotted as a function of continuous orientation shift between background line elements. Different line densities were tested. The thin straight lines in panel $\mathbf{C}$ indicate the target-free conditions $\left(\Delta_{\mathrm{tg}}=\Delta_{\mathrm{bg}}\right)$; the corresponding value in panel $\mathrm{D}$ is $\Delta_{\mathrm{tg}}=0^{\circ}$. The targets pop out when local orientation contrast is sufficiently higher than background variation.

that the visual phenomenon of pop-out is given by $75 \%$ correct target detection.

The subjects performed the task on patterns with three different line densities. The testing conditions for Subjects K.D. and I.H., on the one hand, and Subjects C.H., B.S., and R.Z., on the other, were slightly different. The first 2 subjects (Figure 5C) were tested with the following patterns: (1) a high-density $15 \times 15$ element raster, with a raster width (rw) of $0.48^{\circ}$ (open squares); (2) a mediumdensity $12 \times 12$ element raster with rw $=0.6^{\circ}$ (closed cir- cles)-this arrangement was close to that used in Experiment 1; and (3) a low-density $6 \times 6$ element raster with widely spaced elements with $\mathrm{rw}=1.2^{\circ}$ (open circles). The viewing distance was $220 \mathrm{~cm}$. Subjects C.H., B.S., and R.Z. (Figure 5D) were tested with (1) a high-density $15 \times 15$ element raster with $\mathrm{rw}=0.45^{\circ}$ (open squares); (2) a medium-density $9 \times 9$ element raster with $\mathrm{rw}=0.9^{\circ}$ (closed circles); and (3) a low-denisty $5 \times 5$ element raster with $\mathrm{rw}=1.8^{\circ}$ (open circles). The viewing distance was $203 \mathrm{~cm}$. The line elements had the same size in all of the 
patterns $\left(0.33^{\circ} \times 0.08^{\circ}\right.$ for the data shown in Figure $5 \mathrm{C}$; $0.37^{\circ} \times 0.09^{\circ}$ for the data in Figure 5D). The targets appeared at eccentricities of $0.87^{\circ}-2.33^{\circ}$ (Figure 5C) and $1.8^{\circ}$ (Figure 5D) to either side of the fixation point.

Under all of the tested conditions, the target orientation contrast required for pop-out increased with increases in the overall variation of line orientation in the pattern, in a way very similar to the increase of threshold orientation contrast at texture borders with higher background variation (Experiment 1). This suggests that both pop-out and texture segmentation result from a similar neuronal mechanism, which apparently is based on the detection of local orientation contrast rather than on the analysis of orientation per se. In fact, in many patterns, other line elements had the same orientation as that of the target, but they did not pop out perceptually. As for texture segmentation, no orientation difference was found to pop out from a pattern with sufficiently high background variations $\left(\Delta_{\mathrm{bg}}>30^{\circ}\right)$.

Stimulus patterns used for the curves in Figures $5 \mathrm{C}$ and 5D differed by a small but apparently important property. In the patterns used for Figure 5C, the continuous background orientation shift was interrupted at the target position to make the four neighbors of the target all display the same orientation (e.g., Figure 5B). With such patterns, the threshold orientation contrast was consistently
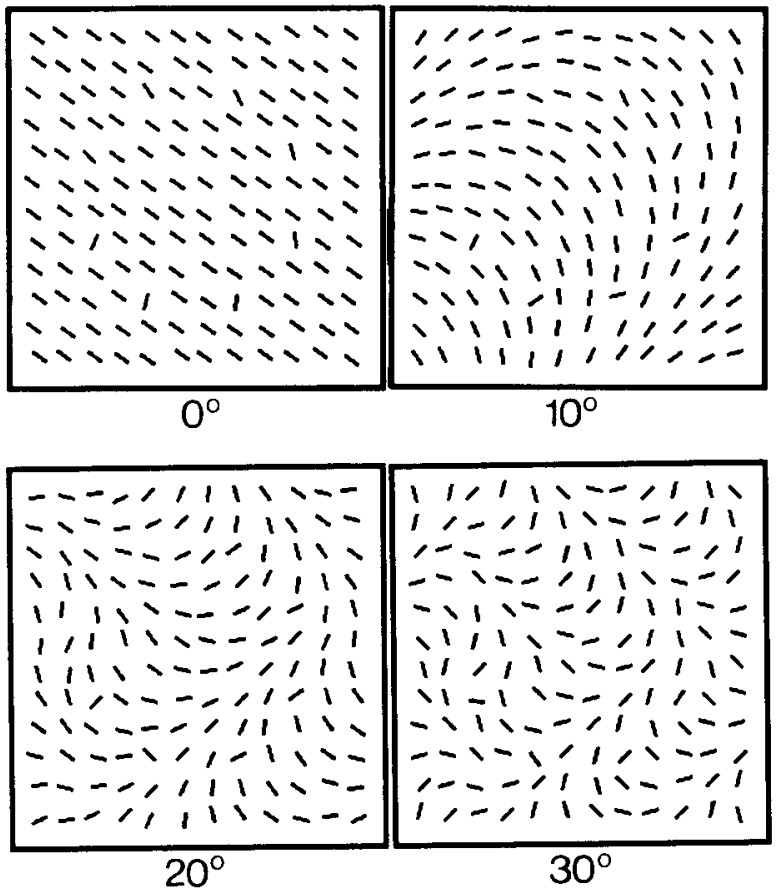

Figure 6. Pop-out depends on background variation. The patterns show eight pop-out targets in a circle-like configuration; their local orientation contrast increases clockwise in steps of $10^{\circ}\left(\Delta_{\mathrm{tg}}=10^{\circ}\right.$, $\left.20^{\circ}, \ldots, \mathbf{8 0}^{\circ}\right)$. For a uniform texture background $\left(\Delta_{b z}=0^{\circ}\right)$, almost all elements can be seen spontaneously. With increasing variation of background elements $\left(\Delta_{\mathrm{bg}}=10^{\circ}, \Delta_{\mathrm{bg}}=20^{\circ}\right)$, fewer elements stand out from the pattern. Almost all of them seem to be hidden when background variation is large enough $\left(\Delta_{\mathrm{bz}}=30^{\circ}\right)$. decreased for widely spaced line textures (Figure 5C, open circles). In the stimulus patterns used for Figure 5D, the texture flow was continuous across the whole pattern, and target orientation contrast was locally added to the line orientation of the background element at the target's position (as in the patterns shown in Figure 6). With these patterns, threshold curves for different line densities were similar and could, in fact, fall almost congruently (Subject C.H.). Note that target orientation contrast, in these patterns, resembles the orientation difference from the continuous flow pattern. Exact orientation differences between the target and its next neighbors may be different from that value; this is particularly true for an orientation contrast of $90^{\circ}$, for which the local orientation difference between the target and its neighbors is only $90^{\circ}-\Delta_{\mathrm{bg}}$. (These differences may be one explanation for the observed difference in threshold curves for patterns with low-density textures; cf. the open-circle curves in Figures 5C and 5D.) On the other hand, construction of patterns as in Figure 5B produces a local patch of similar lines-a figure that itself may be easily detected in low line density patterns. (This is another possible explanation of why sensitivity is increased in low-density textures; cf. the open-circle curves in Figure 5C.)

The effect of the overall variation of line orientation in a pattern on perceived pop-out of target elements is demonstrated in Figure 6, which displays eight target elements in a circle configuration, with clockwise increasing orientation contrast to the background $\left(\Delta_{1 \mathrm{~g}}=10^{\circ}\right.$, $\left.20^{\circ}, 30^{\circ}, \ldots, 80^{\circ}\right)$. Depending on the background orientation shift, almost all $\left(\Delta_{\mathrm{bg}}=0^{\circ}\right)$ or only few $\left(\Delta_{\mathrm{bg}}=30^{\circ}\right)$ targets, if any, can spontaneously be detected. Note that lines elsewhere in the pattern do not pop out even if they share the orientations of salient elements. Thus, orientation per se does not seem to provide pop-out. The role of saliency in searching for a line at a certain orientation was investigated in the following experiment.

\section{Experiment 4: Without Orientation Contrast, Visual Search Is Serial}

In this experiment, subjects were required to detect vertical lines under different stimulus conditions, as salient or nonsalient elements of the texture array. Reaction time (RT) was measured as a function of the number of elements shown. Three stimulus conditions were tested (cf. Figure 7): patterns without salient elements (Condition A), patterns with one salient element that was or was not the target (Condition B), and patterns with different numbers of salient elements, one of which could be the target (Condition C). A texture element was made salient simply by presenting it at an orientation contrast far above the variation of orientation between background elements $\left(\Delta_{\mathrm{bg}}=10^{\circ}, \Delta_{\mathrm{tg}} \geq 60^{\circ}\right)$. The different stimulus conditions, A, B, and C, were tested in separate runs. Vertical lines and lines at close orientations $\left( \pm 14^{\circ}\right)$ were absent from half $(A)$ or one third $(B, C)$ of the stimulus patterns shown (no-target condition).

Figure 7 shows some examples of the stimulus patterns used in this experiment. In Conditions A and B, the num- 
CONDITION A
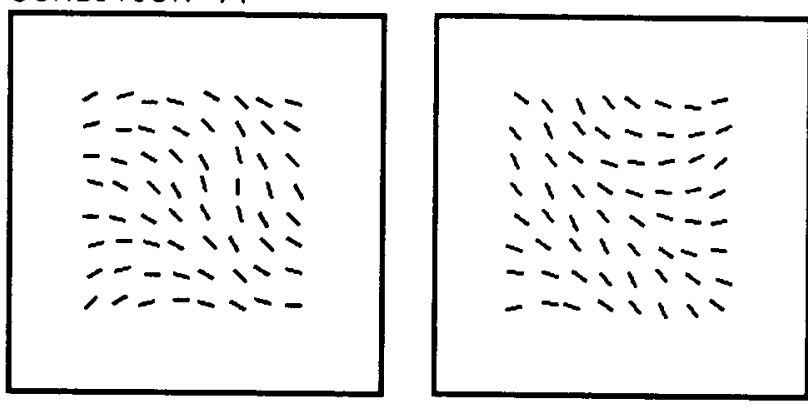

CONDITION B
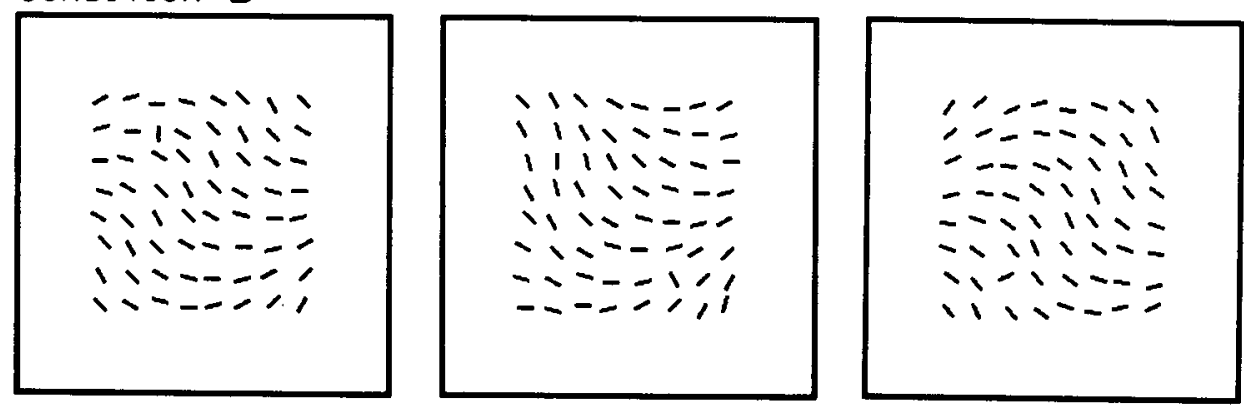

CONDITION C
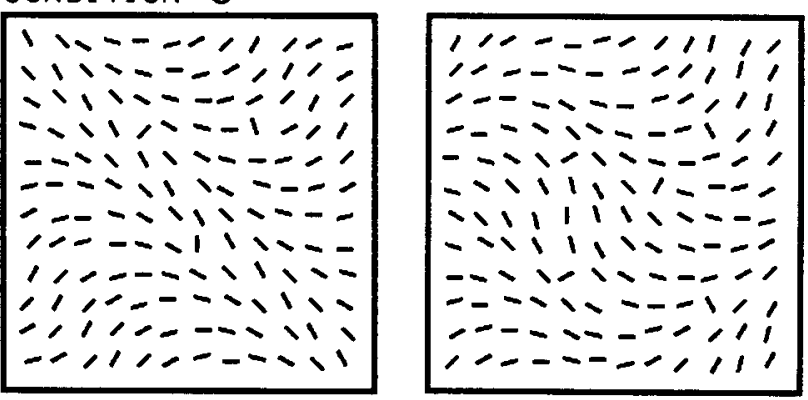

$-\infty 111$

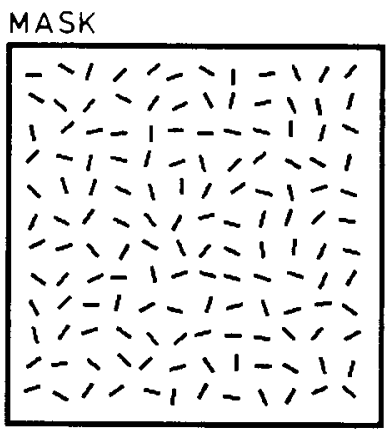

$1,-21$

$-\infty-1$

$-\infty 111$

1110

$1,1,1+2$

$1,0-1$,

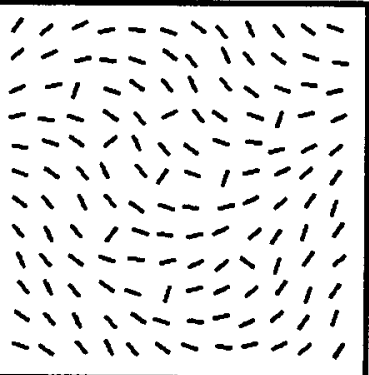

Figure 7. Stimulus patterns used in visual search experiments. The stimulus patterns displayed $4 \times 4,6 \times 6,8 \times 8$ (examples in the top rows), $10 \times 10$ (not shown), or $12 \times 12$ line elements (examples in the bottom row). They were replaced by a mask $(12 \times 12$ elements, top right) immediately after the subject's reaction. The targets (vertical lines) were shown under three different conditions. In Condition $\mathbf{A}$, the targets were nonsalient, with local orientation contrast equal to that elsewhere in the pattern. The examples in the top row show instances of the target (left) and the no-target case (right). In Condition B (middle row), the stimulus patterns always contained an element that had particularly high local orientation contrast and appeared salient among the other line elements. This element could be the target (example on the left) or any other item in the pattern, in which case the target could be either nonsalient (middle) or absent (right). In Condition $C$, the number of salient elements (high local orientation contrast) was varied between 1 and 9. The patterns in the bottom row show examples with 3,5 , and 9 salient elements (from left to right) for cases where the target is one of the salient elements (left), a nonsalient element in the background of the pattern (middle), or not present (right). Targets and salient elements appeared at random positions (but not in the outermost rows and columns of a pattern).

ber of items was varied, and the patterns displayed arrangements of $4 \times 4$ (only in Condition A), $6 \times 6,8 \times 8$, $10 \times 10$, and $12 \times 12$ line elements. In Condition C, all patterns contained $12 \times 12$ lines, and only the number of salient elements was varied. The lines had identical dimensions in all these patterns and were plotted at the same density. The targets could appear at any position in the pattern, except in the outermost rows or columns of the line raster, so that the targets were always surrounded by neighboring lines. (The subjects were not told of this restriction.) In patterns with more than one salient element (Condition $\mathrm{C}$ ), these were never shown in adjacent positions.

In contrast with the previous experiment, in which subjects had to indicate the position of a particularly salient target, here they were asked to indicate the presence or absence of a certain target (vertical line), ignoring its position. They were instructed to perform this task accurately 

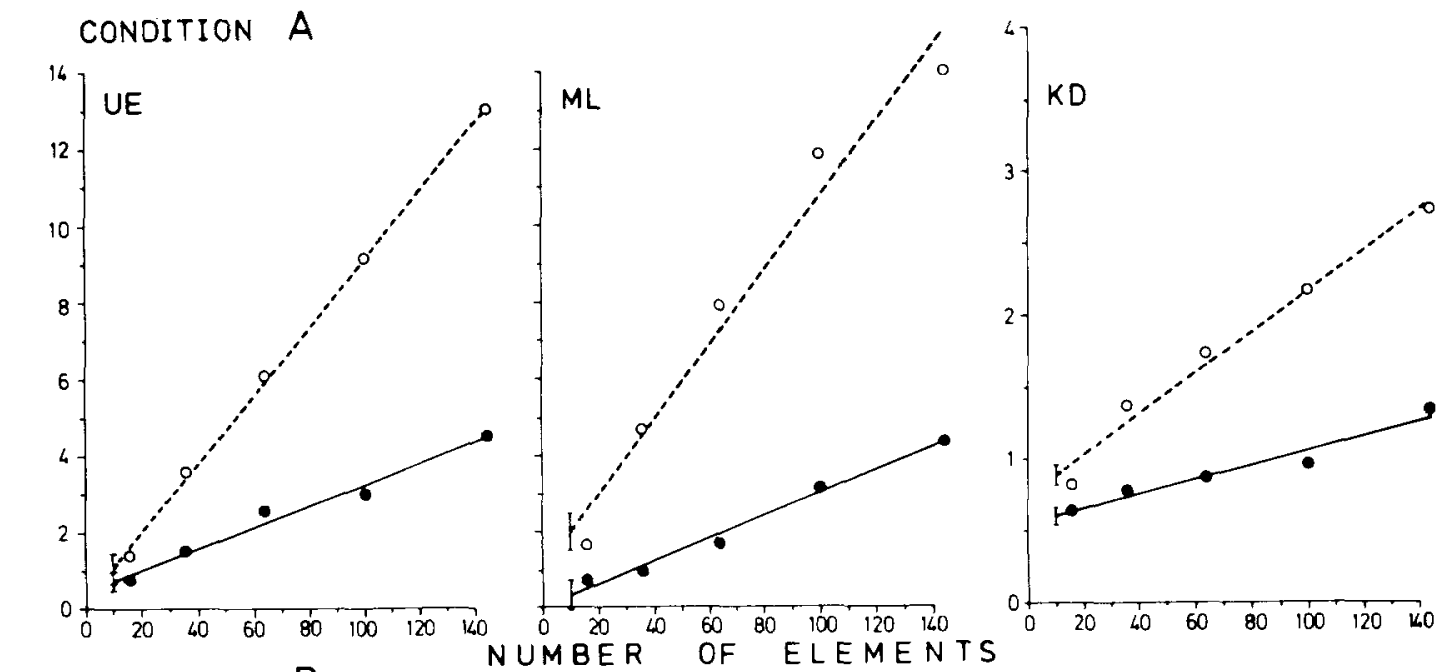

- CONDITION B
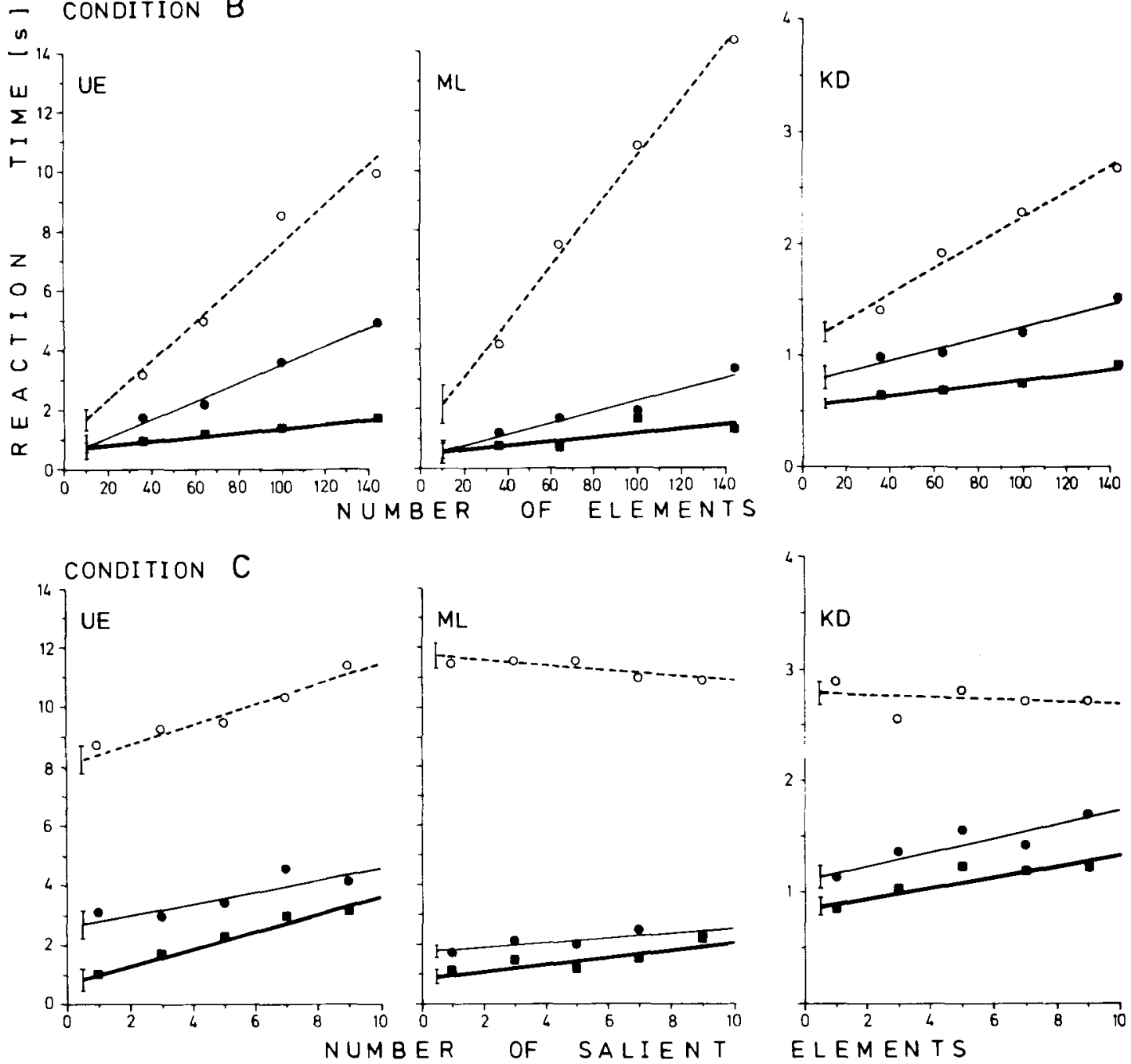

NUMBER

OF SALIENT

ELEMEN T S

Figure 8. Fast and slow visual search for vertical lines. Mean RTs are given for 3 subjects (U.E., M.L., and K.D.) in Conditions A-C. Mean RTs for detecting salient targets are plotted by filled squares (thick continuous lines); RTs for nonsalient targets by filled circles (thin continuous lines); and RTs for pattern rejection (no target presentations) by open circles (dashed lines). 
Table 1

Reaction Time Measurements of Experiment 4

\begin{tabular}{|c|c|c|c|c|c|c|}
\hline \multirow[b]{3}{*}{ Target } & \multicolumn{6}{|c|}{ Slope of Regression Lines [Milliseconds/Element] } \\
\hline & \multicolumn{2}{|c|}{ Subject U.E. } & \multicolumn{2}{|c|}{ Subject M.L. } & \multicolumn{2}{|c|}{ Subject K.D. } \\
\hline & Slope & $n$ & Slope & $n$ & Slope & $n$ \\
\hline \multicolumn{7}{|c|}{ Condition A } \\
\hline $\begin{array}{l}\text { Nonsalient } \\
\text { None }\end{array}$ & $\begin{array}{l}27.8 \pm 3.0 \\
89.3 \pm 4.4\end{array}$ & $\begin{array}{l}243 \\
248\end{array}$ & $\begin{array}{l}29.5 \pm 4.6 \\
96.6 \pm 5.6\end{array}$ & $\begin{array}{l}200 \\
199\end{array}$ & $\begin{array}{r}5.0 \pm 0.7 \\
14.2 \pm 0.8\end{array}$ & $\begin{array}{l}147 \\
150\end{array}$ \\
\hline \multicolumn{7}{|c|}{ Condition B } \\
\hline $\begin{array}{l}\text { Salient } \\
\text { Nonsalient } \\
\text { None }\end{array}$ & $\begin{array}{r}7.0 \pm 1.7 \\
30.5 \pm 4.3 \\
65.4 \pm 3.9\end{array}$ & $\begin{array}{l}200 \\
190 \\
199\end{array}$ & $\begin{array}{c}7.1 \pm 2.7^{*} \\
19.2 \pm 3.9 \\
92.9 \pm 6.9\end{array}$ & $\begin{array}{l}160 \\
160 \\
160\end{array}$ & $\begin{array}{r}2.3 \pm 0.5 \\
5.0 \pm 1.1 \\
11.3 \pm 0.9\end{array}$ & $\begin{array}{l}159 \\
155 \\
160\end{array}$ \\
\hline \multicolumn{7}{|c|}{ Condition $\mathrm{C}$} \\
\hline $\begin{array}{l}\text { Salient } \\
\text { Nonsalient } \\
\text { None }\end{array}$ & $\begin{array}{l}276.6 \pm 65.7 \\
186.3 \pm 82.8 \dagger \\
317.6 \pm 81.7\end{array}$ & $\begin{array}{l}194 \\
195 \\
200\end{array}$ & $\begin{array}{r}115.5 \pm 40.2^{*} \\
74.0 \pm 34.8 \dagger \\
-85.3 \pm 73.4 \dagger\end{array}$ & $\begin{array}{l}242 \\
249 \\
249\end{array}$ & $\begin{array}{r}45.5 \pm 14.0^{*} \\
59.9 \pm 18.1^{*} \\
-9.8 \pm 18.3 \dagger\end{array}$ & $\begin{array}{l}142 \\
140 \\
150\end{array}$ \\
\hline
\end{tabular}

Note $-n=$ number of stimulus presentations. Differences of slopes from zero are highly significant $(p<.001)$, except where marked $\left({ }^{*} p<.01 ; \dagger p<.05\right)$.

and as fast as possible. The stimulus patterns were shown continuously until the subject pressed a "ready" button to indicate that a decision was made. This time was taken as the subject's RT. The stimulus pattern was then immediately replaced by a mask, and the subjects could enter their responses (vertical line present or absent) as fast or as slowly as they wished. Mask patterns displayed $12 \times 12$ lines at random orientations (including vertical ones) and remained visible until the subject had responded. After a break of about $3 \mathrm{sec}$, a new trial was started. Note that in this procedure, RTs for hits and rejections were both taken from identical actions of the subject. Different responses as to the presence or absence of vertical lines were distinguished from subsequent typing when the RT had already been measured.

In Figure 8, the mean RTs of 3 subjects are plotted against the number of line elements (Conditions $A$ and B) or pop-out targets (Condition C) in the pattern. RTs were averaged for correct responses (see Table 1). False alarms were rare $(<0.7 \%$; see Table 2$)$, but vertical lines were sometimes missed, in particular when they were nonsalient (i.e., when they did not display local orientation contrast).

The data in Condition A were obtained with patterns from which salient line elements were completely absent. The vertical lines did not pop out from these patterns, and subjects reported that they had to look carefully for them. This is reflected in a considerable increase of RT from

Table 2

Errors in Detecting Vertical Line Elements (Experiment 4)

\begin{tabular}{lccc}
\hline & \multicolumn{3}{c}{ Subject } \\
\cline { 2 - 4 } & U.E. & M.L. & K.D. \\
\hline False alarms & $0.7 \%$ & $0.6 \%$ & $0.0 \%$ \\
Misses of salient targets & $1.5 \%$ & $1.7 \%$ & $2.9 \%$ \\
Misses of nonsalient target & $4.7 \%$ & $4.7 \%$ & $2.6 \%$ \\
\hline
\end{tabular}

Note-In Condition B, Subject K.D. missed only $0.6 \%$ of salient targets and Subjects U.E. and M.L. missed no salient targets at all. the 16-item to the 144-item presentations; slopes (see Table 1) were about $30 \mathrm{msec} /$ item for Subjects U.E. and M.L., and $5 \mathrm{msec} / \mathrm{item}$ for Subject K.D. Mean RTs for correct rejections (i.e., correct identifications of patterns from which vertical lines were missing) increased with slopes of about $90 \mathrm{msec} /$ item (14 msec/item for Subject K.D.). Note that these values consistently exceeded what one would expect from the characteristics of serial search, where RT for no-target presentations should be about double that for target presentations. However, remember that targets were excluded from positions in the outermost rows and columns, in order that they be always surrounded by neighboring elements. Therefore, if subjects were following a search strategy of moving from central parts of the pattern to more peripheral ones, they would never need to screen as many elements in the target as in the no-target presentation. The possible underestimation of the slopes in target presentations is about $25 \%$. If values were accordingly corrected (multiplied by 1.3), the relationship of slopes in target and no-target presentations would more closely fit the expectations. Also, subjects missed some of the nonsalient targets; the estimates of their mean RT might be slightly too low. However, both the pronounced increase of RT with an increasing number of displayed elements and (roughly) the doubling of RT for no-target presentations are clear indications of serial search for vertical lines in these patterns.

The curves for Condition B plot the data for patterns with one salient line element. When this salient line was not the target for which subjects were looking (filled circles), they had to search serially through all the background elements in order to detect the vertical line, similarly to the situation when salient lines were completely absent (Condition A; filled circles). However, when the salient line element happened to be a vertical line (squares), subjects detected that target immediately. Mean RTs were greatly reduced in this case and showed less variation with the number of items in the pattern (the slopes were about $7 \mathrm{msec} /$ item for Subjects U.E. and 
M.L. and $2.3 \mathrm{msec} / \mathrm{item}$ for Subject K.D.), which is assumed to indicate parallel search.

In the third test (Condition C), texture patterns contained different numbers of salient elements, the total number of texture elements being constant in these patterns $(n=144)$. In this condition, RT increased linearly with the number of salient elements whether the target was among the salient (squares) or the background (filled circles) elements. Mean RT was, in general, much longer for patterns from which the target was missing (open circles), and there was no consistent variation between subjects with the number of salient elements in this case. Note that deviations of slopes from zero were nonsignificant for Subjects M.L. and K.D. (Table 1).

Analysis of the standard deviations (SDs) of the RTs revealed a linear increase with increases in sample size for the nonsalient target presentations of Conditions $A$ and $B$ and a linear increase with increases in the number of salient elements for salient targets in Condition C, where search was assumed to be serial. SDs for nonsalient or no-target presentations in Condition $\mathrm{C}$ were almost constant and independent of the number of salient elements presented.

Mean RTs were, in general, increased for nonsalient versus salient targets, indicating that, even in Condition $C$, saliency of the target helped to detect it. The fact that detecting a nonsalient target in patterns with one salient element (Figure 8B, filled circles) took only about as long as did detecting a salient target among nine salient elements (Figure 8C, filled circles) suggests that even for nonsalient elements search is not done element by element. This could be due to the fact that line elements in these patterns varied systematically in line orientation. Search for vertical lines, even when not popping out from the pattern, could probably be made by inspecting patches of line elements rather than single elements. Such a strategy may also explain why the slopes of the RTs for missing targets were not exactly double those for nonsalient targets (Conditions $\mathrm{A}$ and $\mathrm{B}$ ), an observation also made by Wolfe, Friedman-Hill, Stewart, and O'Connell (1992).

An interesting observation is the variation in RT among Subjects U.E., M.L., and K.D. (Table 1). This also corresponds to observations by Wolfe et al. (1992), who noted large variations of slopes in search tasks between subjects. Subject K.D. of the present study, who was highly trained in texture segmentation and pop-out tasks, might have adopted a particularly fast strategy in searching for vertical lines. However, despite the fact that she reacted much faster in these tests than Subjects U.E. or M.L., her RT data show the same characteristic slope differences for the nonsalient, salient, and no-target conditions.

Altogether, these curves show that detecting a vertical line could only be considered to resemble a parallel process when the vertical line itself was a salient, and preferably the only salient, element of the pattern. In this situation, RT variation with the number of line elements was minimal. Both when no lines popped out and when pop-out was associated with other line orientations, RTs strongly increased with the number of displayed items, suggesting a serial process in searching for the vertical line. This provides strong evidence against the notion that features are preattentively detected and recognized (Treisman, 1985, 1986; but see Treisman, 1988).

An indication of these results can be obtained from inspection of Figure 7. Detection of the target, a vertical line, is easier and can apparently be done faster when the target is salient, because of local orientation contrast (Condition $B$, on the left), than when the target is not more salient than other elements in the pattern (Condition $A$, on the left; or Condition B, in the middle).

The experiments of this section have shown that popout of orientation, like texture segmentation, is based on orientation contrast but not on oriention itself. The fact that a sufficiently large local orientation contrast is a sufficient (Experiment 3) but also necessary (Experiment 4) key for fast visual search argues against the special role of orientation features in preattentive vision. Furthermore, the detection of a specific target was shown to resemble a serial process if the target was not sufficiently distinct from its neighbors.

Altogether, the results from these experiments agree with the view that the fast (and hence, presumably, preattentive) detection of orientation features is based on the preattentive detection of local dissimilarities and the attentive (and apparently serial) identification of the features themselves (cf. Sagi \& Julesz, 1985). This does not exclude the possibility that categorical aspects and topdown processes may provide extremely fast (and perhaps parallel) search among items that are locally distinct (Wolfe et al., 1992; see the Discussion section below).

\section{TWO COMPONENTS IN PREATTENTIVE GROUPING}

The important role of feature contrast in both texture segmentation and pop-out, and the unimportance of features themselves, raises questions about their role in grouping phenomena. Is grouping based on similarity or on saliency? That is, do elements of a pattern perceptually group because they are identical, or because they all are sufficiently distinct from their next neighbors? Subjects detect the circle of salient elements in Figure 6 well, despite the fact that the orientations of the line elements are different. This suggests that line orientation does not affect perceptual grouping. On the other hand, however, the orientation of line elements itself is not ignored, and observers can selectively switch between lines at different orientations grouping together. This is illustrated in Figure 9. The first view shows a circular configuration of six salient elements, which arises from the local dissimilarity of these line elements that is independent of their actual orientations. By concentrating on lines of a certain orientation, one can see one of the two triangles that are formed from identical lines. Thus, the information on line orientation-the feature itself-can be used to modify the 


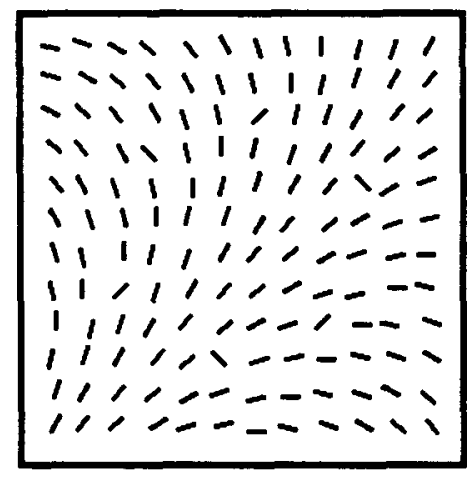

Figure 9. Preattentive and attentive grouping. The pattern shows six salient elements in a circle-like configuration. By concentrating on line elements of a certain orientation, two subgroups of these elements can be distinguished, each of which is made from identical lines. What is the instantaneous percept of this figure? Do we see triangles, because of the similarity of elements, or do we see a circle, because of their local orientation contrast?

grouping process. However, even concentration on a certain orientation-for example, on vertical lines-cannot produce the percept of nonsalient line elements forming a separate group. This indicates that saliency from local orientation contrast is a prerequisite for grouping.

It seems likely that grouping for feature similarity (e.g., seeing the global triangles in Figure 9) is based on a secondary step of analysis that may well be influenced by attention. Sagi and Julesz (1985) have shown that figural grouping of salient elements can occur without knowledge of the individual elements' orientations. Also, when one looks at Figure 1, one's first impression is that it consists of a circle and not of triangles intersecting each other, an impression that is achieved only during prolonged inspection. The fact that an observer can voluntarily switch between groups of different line orientations suggests that higher, probably top-down processes are involved, the investigation of which lies outside the scope of this study on preattentive vision. The role of similarity in preattentive grouping was investigated in the following experiments.

\section{Experiment 5: Preattentive Grouping}

\section{Is Independent of Element Similarity}

Subjects were asked to detect groups of elements within a texture array and to distinguish global configurations formed by them.

The configurations were triangles made of salient elements. The triangles could point upward, downward, to the left, or to the right (see Figure 10). The triangles were made of similar (Condition A) or dissimilar line elements (Condition B), all of which had the same local orientation contrast with respect to their neighbors (Figures 10A and $10 B$ ). Within a run, the local orientation contrast of target elements was systematically varied in order to measure differences in detection rate between these conditions. Without the subjects' being told, stimulus patterns from a third condition $(C)$ were intermixed into the run. In these patterns (Figure 10C), triangles of similar lines were shown together with an additional but dissimilar line element at a position opposite to the triangle's pointer, so that salient line elements formed the figure of a square rather than a triangle. If similarity played an important role for preattentive grouping, one should expect subjects to respond better (i.e., with lower threshold) to triangles made of similar line elements than to triangles made of dissimilar lines. They also should easily detect the triangle of similar elements in Condition $\mathrm{C}$, which was taken as the "correct" response for this condition. However, if grouping were based on the saliency of target elements alone and information on line orientation, and if the similarity of elements were ignored, performances in Conditions $A$ and $B$ should be similar and subjects should respond at chance level in Condition $C$.

The stimulus patterns were shown for $160 \mathrm{msec}$ and masked afterwards. Actual line orientation was randomly varied between presentations. Three subjects were tested; their data are shown in Figure 10D. The open symbols refer to true triangle stimuli with similar (continuous curves) or dissimilar target elements (dashed curves). With increasing target orientation contrast, the detection of triangles increased. The closed symbols refer to tests in which four salient elements were present, and in which subjects never performed much better than chance (indicated by a thin straight line). Note that Subject K.D. was only tested with triangles pointing upward or downward (i.e., chance was at $50 \%$ ), whereas Subjects C.A. and I.H. were both tested with triangles pointing in any of the four directions (chance was at $25 \%$ ). The detection rates for Conditions $A$ and $B$ (triangles with similar and dissimilar elements, respectively) show only small differences, indicating little or no contribution from similarity aspects in this task. This is confirmed by the data of Condition $\mathrm{C}$, which show that subjects did not prefer the figure of identical lines over figures from any combination of salient elements. It should be stressed that voluntary selection of a certain line orientation does allow identification of the triangle of similar lines in Condition $\mathrm{C}$. However, such a selection would have required much longer presentation times for the stimulus patterns than those used here.

\section{Experiment 6: Alignment Efrects in Configurational Grouping}

The following experiment was performed to test whether, in grouping, there would be interactions between line orientation and figural form that would be similar to those found with texture segmentation (Experiment 2). Two stimulus conditions were compared, in which salient line elements in a circle-like configuration were oriented either tangentially or radially to the outline of the circle. The background variation of line orientation was adjusted to produce various degrees of visibility of the circle figures in these patterns (Figures 11 A and 11B).

The displays were presented for $100 \mathrm{msec}$. The subjects were required to indicate whether or not the circle 
A

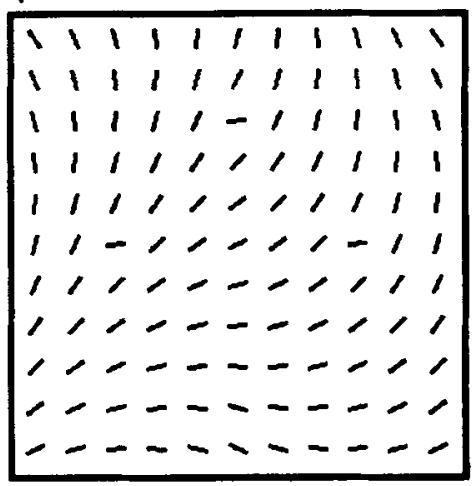

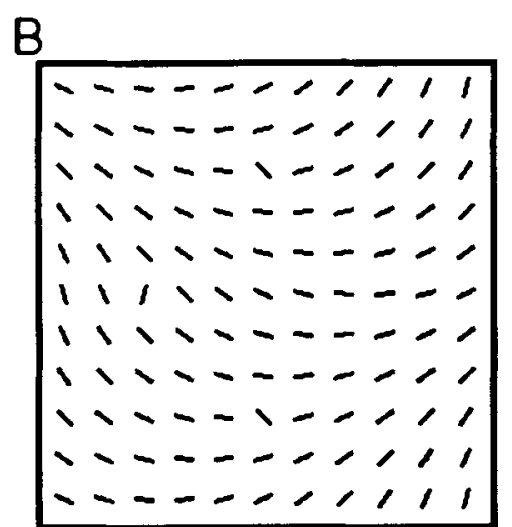

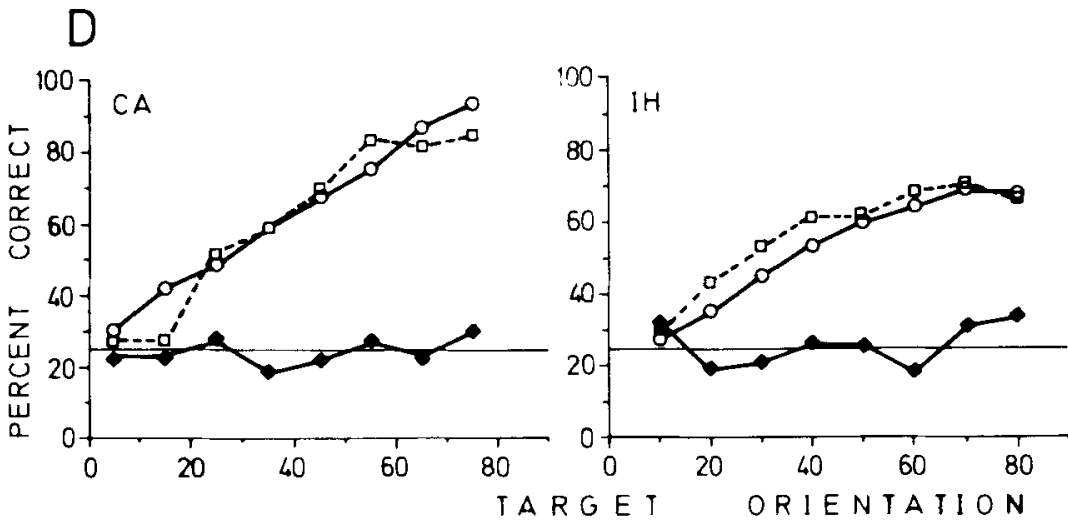

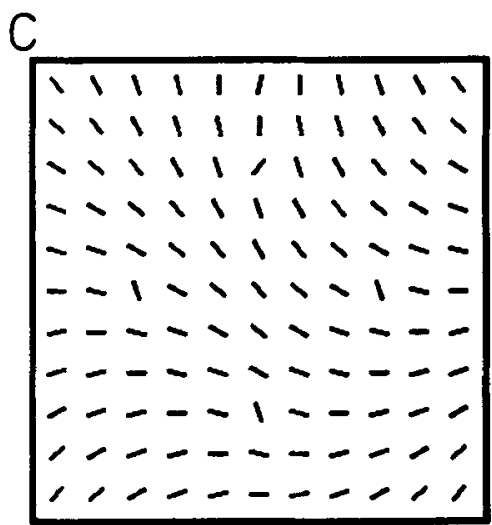

Figure 10. Preattentive grouping is independent of element similarity. From brief inspection of the pattern, subjects had to detect the global configuration of salient elements that formed triangles pointing in different directions. Of these elements, either all were identical (A) or one was different (B), but all displayed the same local orientation contrast. Subjects were not informed of this, but patterns from a third condition (C) were intermixed into these runs, in which groups of four elements (three same, one different) were present. The open symbols in D give the detection rates (as functions of the local orientation contrast of target elements) for 3 subjects responding to triangles of similar (continuous curves) and dissimilar lines (dashed curves). The filled symbols plot the detection rates for "same" triangles in four-point configurations (taken as the "correct" response in these patterns). Detection rates for the true triangles are very similar, independent of target identities. Detection rates from four-point configurations remained at chance (thin line). For Subject K.D., only triangles pointing upward or downward were tested (chance level 50\%).

was present in the pattern shown. As a control, patterns with random distributions of the same line elements also were shown. These patterns did not contain the circle figure. Figure $11 \mathrm{C}$ shows the circle detection rates of 3 subjects for the patterns shown in Figures 11A and 11B. Note that for patterns with moderately visible figures (in the second and third columns), all subjects preferred the tangential over the radial configuration; that is, they more often detected the circle when the orientation of line elements was aligned with the circle's outline than when elements were orthogonal to it. In a few patterns (third and fourth columns), some subjects could detect the circle even from radial configurations of line elements, suggesting that the percept was not exclusively due to the alignment of lines and figure outlines but that saliency itself was an important factor in the detection of the circle. This underlines the limited influence of alignment effects in configuration grouping. They were dominant only when the saliency of target elements was close to threshold (cf. Experiment 2C). If line elements were nonsalient, neither the aligned (tangential) nor the nonaligned (radial) versions of the stimulus were detected (left-hand columns in Figure 11C). With highly salient elements (fourth column), on the other hand, both versions could produce similar detection rates (Subject C.L.).

Perceptual grouping, in our experiments, was found to be based primarily on the saliency of line elements, which is both necessary and sufficient for the preattentive percept of groups of elements to stand out from the pattern. Such groups neither include nonsalient elements nor, in preattentive vision, would form subgroups of identical elements. Such subgroups can, however, be seen under extended viewing conditions.

When one is looking for global configurations of salient elements, the alignment of individual elements with the outline of a figure may help it to be seen. This effect is qualitatively similar to that seen for texture segmentation (Figures 2 and 3), and it seems to play an important role only for patterns in which the saliency of the figure is near threshold. 

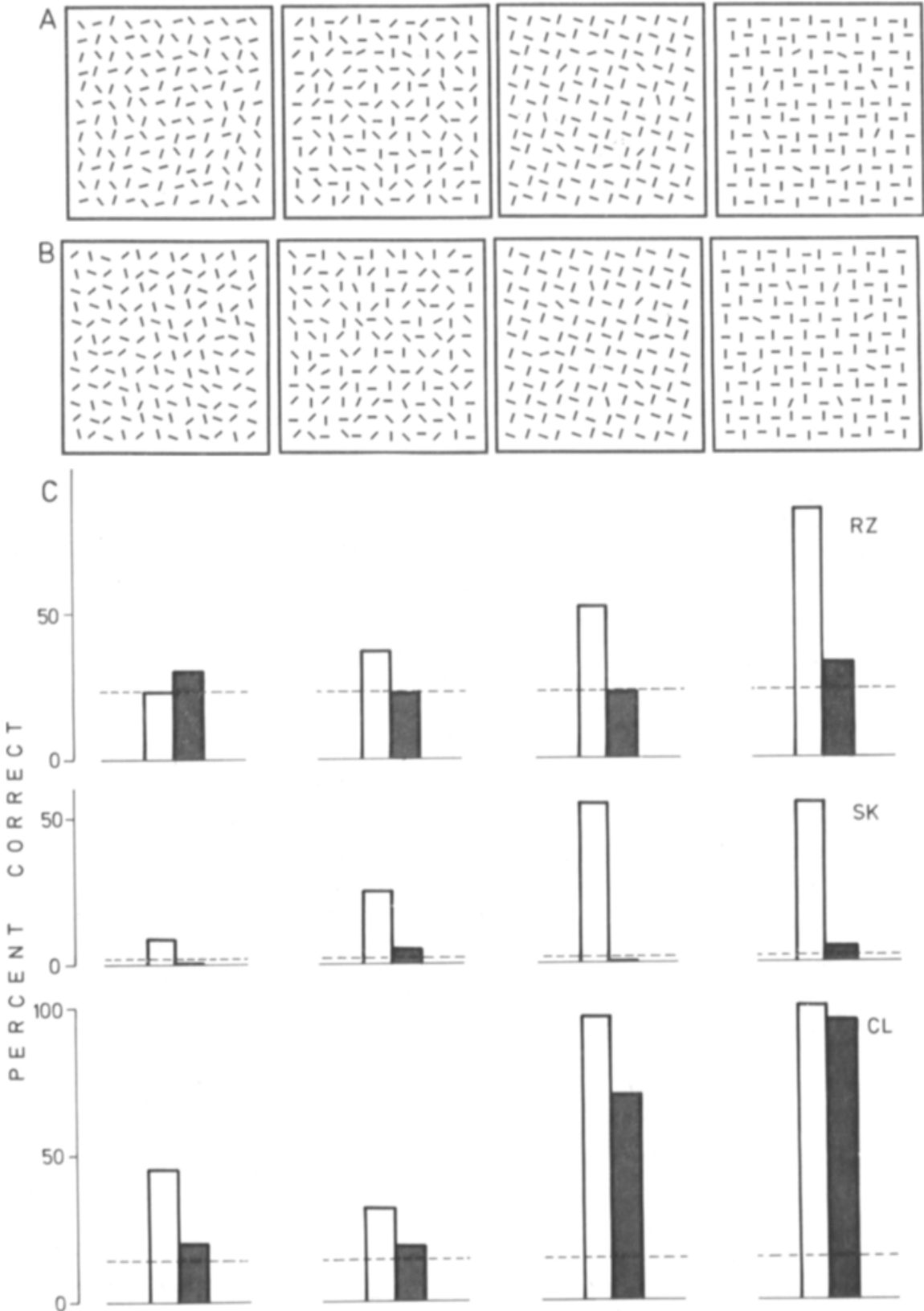

Figure 11. Alignment effects in grouping. Rows $A$ and B present stimulus patterns with popout elements in the configuration of a circle. Line elements are oriented tangentially $(A)$ or radially (B) to the outline of the figure. The patterns were constructed to display easily visible (patterns on the right side) or barely visible (patterns on the left side) circles for tangential configurations. The histograms (C) show the detection rates for 3 subjects responding to patterns as in row A when lines were aligned with the circle (open bars), or perpendicular to it (filled bars). The percentage of false alarms to control patterns is marked by a dashed line. With increasing visibility of the circle configuration, the subjects' detection rates increased. For most conditions, however, the aligned versions of the patterns were seen more often than the nonaligned versions. 


\section{DISCUSSION}

\section{Feature Analysis in Preattentive Vision}

Three phenomena of preattentive visual perception were studied in this paper. They all were shown to depend, in a very similar way, on local dissimilarity in the line pattern (i.e., feature contrast), rather than on global analysis of line orientation (i.e., the feature itself). Texture areas were seen as units even when they were made of line elements at quite different orientations, and subjects detected groups of dissimilar elements as well as groups of similar elements, often without even noting the difference. These observations provide strong evidence against a major role of feature analysis in preattentive vision. Features themselves were not easily detected unless they were particularly salient (Experiment 4), and saliency was found to depend not on features but on feature contrast. It was shown that the saliency of texture elements is due to local dissimilarities in line orientation and is globally influenced by the overall orientation variation within a pattern. As discussed elsewhere (Nothdurft, 1991c), this suggests a mechanism continuously dependent on orientation contrast, which has been shown to be a prominent response property of $\mathrm{V} 1$ and $\mathrm{V} 2$ cells in the macaque monkey (Knierim \& Van Essen, 1992; Van Essen et al., 1989).

Although such a conclusion is convincing with regard to the stimuli tested in this study, other cues besides orientation can induce segmentation of line arrays. Of course, when line elements are arranged in such a way that texture areas differ in spatial frequency composition (Hallett \& Hofmann, 1991), segmentation can be strong, independently of the local orientation contrast of line elements at the texture border. In fact, such patterns segment even when made small enough so that the orientation of line elements cannot be seen. It is not yet clear to me, however, whether segmentation without a particular orientation contrast is always due to such effects alone. The lines forming the circle in Figures 11A-11B did not, in fact, display any larger local orientation contrast than did the lines in the background. Nevertheless, the circle can easily be seen, particularly in the right-hand configurations of Figure $11 \mathrm{~A}$, where background elements are alternatingly vertical and horizontal. It is less visible when background elements are slightly rotated (Figure 11A, third column), and detectability becomes poor if other lines are added to the background (which should, in fact, decrease the local variation in background orientation). Whether these effects could be explained by assuming a larger area size over which orientation contrast is evaluated or by differences in spatial frequency composition due to the regularity of the background pattern remains to be investigated.

The observations in the present study are also interesting with regard to findings from other studies. Moraglia (1989), in studying the detectability of horizontal lines, found that targets interrupting a continuous flow pattern were detected much faster than were targets fitted into the global line flow (low target orientation contrast) or targets embedded in a random line pattern (large background variation in terms of the present study). These observations are similar to those obtained in Experiments 3 and 4 of the present study. Also, from search experiments, Duncan and Humphreys (1989) have concluded that speed of visual search is related to the dissimilarity between the target and nontargets and to similarities among nontargets. Such a relationship is also reflected in the data from the present study. For increasing background variation (decreasing similarity among nontargets), target orientation contrast (target-nontarget dissimilarity) had to be increased for pop-out to be obtained (see Figure 5). The important aspect added in the present study was the role of local dissimilarities, in contrast to the global similarity analysis in Ducan and Humphreys's approach. In fact, pop-out occurred achieved even with targets that also were members of the group of nontargets (see Figure 6), provided that targets and nontargets were locally dissimilar.

\section{Local Analysis of Texture Gradients}

The ability to detect texture borders has also been found to be based on the local analysis of differences-that is, on the detection of texture gradients. Whereas in earlier studies, researchers mainly discussed the role of element density and interelement separation (Julesz, 1986; Nothdurft, 1985b), the present study underlines the important role of feature (orientation) gradients even for constant element spacing (see also Nothdurft, 1985b, 1990, 1991c). Bacon and Egeth (1991) have recently called into question the importance of local processing for fast visual search. When searching for vertical or horizontal lines among horizontal or vertical distractors, they did not find any variation in $\mathrm{RT}$ with line spacings of up to $4^{\circ}-5.6^{\circ}$, whereas other investigators have found a reduction in performance, in both detection (Sagi \& Julesz, 1987) and segmentation tasks (Nothdurft, 1985b), for such widely spaced elements. However, Bacon and Egeth (1991) have found some variation in RT with the number of line elements in the display, which they discussed with regard to similarity grouping of distractors increasing search efficiency (Duncan \& Humpreys, 1989). One could argue that their observations are consistent with the notion that saliency, and not orientation per se, provides fast visual search. Note that for sparse arrangements of lines around the target and (because of the large number of displayed elements) denser arrangements among the distractors, targets may become salient from other (e.g., luminance) inhomogeneities in the pattern. Apparently, any saliency would help to accelerate visual search (Nothdurft, in press).

An interesting phenomenon in visual search has recently been added by Wolfe et al.'s (1992) finding of categorical components in the search for oriented lines (see also Treisman \& Gormican, 1988). These authors found that search efficiency is improved if target lines display categorical aspects such as "steep" or "left-tilted," which are absent from the pool of distractors. This seems to contradict the statement made in the present study that fast 
visual search requires sufficient target orientation contrast, independently of the target's orientation. Note, however, that in all stimulus patterns of Wolfe et al.'s study (except those for Experiments 1 and 2) elements displayed a local orientation contrast of $30^{\circ}$ or more and hence were, according to the present study, sufficiently distinct from their neighbors. Whether, under such a condition, topdown processes may improve search strategy or whether the observations may also in part be explained by contributions from neuronal filters with large receptive fields, together with some degree of orientation anisotropy (see Foster \& Ward, 1991a, 1991b), remains open. Interestingly, Wolfe et al. also found that vertical lines presented in a continuum of line orientations (distractors at $20^{\circ}, 40^{\circ}$, $60^{\circ}$, or $80^{\circ}$ to either side of the vertical, $0^{\circ}$ ) required serial search, as in Experiment 4 of the present study. Also in their study, the slopes for target-present and targetabsent trials deviated from a $1: 2$ relationship (19.7 msec/ item: $49.5 \mathrm{msec} /$ item) as they have in my experiments (Table 1).

\section{Target Localization}

I want to explicitly underline the observation implicitly made in the present study that subjects never reported any difficulty in localizing a target when they had detected it. This was the case both in "odd man out" tasks (Experiment 3) and in the vertical line search task (Experimental 4), although in the latter I have not explicitly measured subjects' performances in target localization. This is different from the assumption that target features but not target positions are recognized preattentively (Treisman, 1985). We made similar observations in a recent study in which we triggered eye movements by using local differences in line orientation (Parlitz \& Nothdurft, 1990).

\section{Alignment Effects}

In addition to local orientation contrast, there was notable contribution to the detection of texture borders or global figures from line elements having orientations similar to those of the outlines of the figure to be detected. Such an alignment effect, which was pronounced only for detection rates near threshold, has recently been shown to facilitate the detection of a straight line from exactly aligned squares (Beck et al., 1989). The neural basis of such an effect is not yet clear. It could reflect interactions between neurones with similar orientation tuning (Gilbert, 1985; Gilbert \& Wiesel, 1981, 1989; Mitchinson \& Crick, 1982), which may play an important role in the perception of line continuation. Alignment effects could also be due to the integration of orientation information at higher levels. For vertical bars from vertical line elements, for example, not only the increased responses to local orientation contrast along the texture border but also the responses to vertical line elements may contribute to the neuronal representation of the texture bar, whereas for vertical bars from horizontal line elements, these different contributions would be linked to orthogonal orientations. If both mechanisms add together, as would be the case when line and border orientations are aligned, the signal-to-noise ratio of border representation could be notably better than in either case of one mechanism alone representing the texture border. This interpretation is supported by the fact that texture borders appear to follow the position of aligned line elements, which could lead to considerable distortions of the shape of a texture area (Figure 12). Whether neurones that distinguish the orientation of texture borders exist, and whether their responses to borders from aligned elements are stronger than those to borders from nonaligned elements, remains to be shown. From the studies of von der Heydt, Peterhans, and Baumgartner (1984) on illusory contours, it seems likely that V2 neurones could react in the assumed way.

\section{Is Saliency Based on Orientation Contrast or on Discontinuities in the Flow Pattern?}

This suggests another possibility of how texture segmentation and pop-out may be achieved in the visual system. Borders or targets may simply be seen as places where the continuous line pattern is interrupted (Link \& Zucker, 1987; Or \& Zucker, 1989). Note that even in patterns with a nonzero background orientation shift, the line elements appear to line up, forming global "stripes" of a certain curvature. Texture borders, or salient elements, are often detected exactly at the places where this continuous pattern is interrupted. The fact that it is difficult to achieve saliency from any orientation contrast for background variations larger than $30^{\circ}$ could then be attributed to the limited ability of the visual system to detect line continuations for such large orientation differences.

I admit that such an interpretation has certain advantages. Flow patterns were prominent even in the patterns used in the present study, and they did appear to be distorted with large background variations (see Figure 6), when pop-out also broke down. The observation of Moraglia (1989) that horizontal targets are readily detected

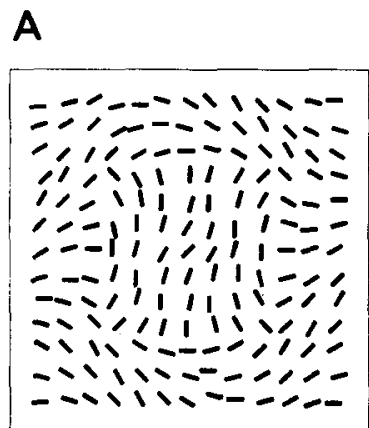

B

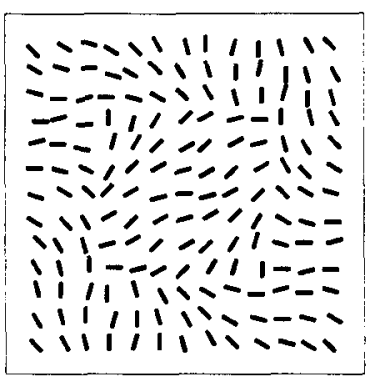

Figure 12. Local line orientation affecting the perceived course of a texture border. Depending on the actual orientation of lines near the texture border, the same stimulus condition $\left(\Delta_{\mathrm{tb}}=90^{\circ}\right.$, $\Delta_{\mathrm{bz}}=15^{\circ}$ ) produces figures of slightly different form. Due to vertical and horizontal lines near its outlines, the square in panel $A$ looks barrel shaped (cf. Nothdurf, 1991) and quite different from that in panel B. Note, however, that the only difference between them is a shitt in line orientation by $45^{\circ}$. 
when they interrupt but not when they continue a circular flow pattern also supports such a view. However, although both notions, orientation contrast and poor continuation, are indistinguishable with many patterns, some of the observations from this study favor orientation contrast. First, large target or border orientation contrasts produce pop-out and the percept of texture borders even in patterns with a $20^{\circ}$ background orientation shift. Thus, if saliency were based on the detection of line discontinuities, $20^{\circ}$ differences should still be seen as continuous and therefore nonsalient. But why, then, do local orientation differences of $20^{\circ}$ pop out in patterns with zero background variation? Second, if saliency were based on the detection of deviations from good continuation, not only larger orientation differences but also those smaller than the background shift should pop out. However, this was not observed. Lines that had, for example, zero contrast with their neighbors were seen as continuation of the global flow pattern, and they did not pop out even when the background orientation varied by $25^{\circ}$ between neighboring positions elsewhere in the pattern. Third, pop-out was not only observed when the assumed continuation of lines was interrupted-that is, with texture borders orthogonal to the orientation of line elements-but also seen between lines with an orientation similar to that of the texture border, which, in fact, lined up with the global flow pattern rather than interrupted it (cf. Figures $2 \mathrm{~A}$ and 2B). This corresponds to neurophysiological recordings from monkey $\mathrm{V} 1$, in which better responses to orientation contrast were not found to be exclusively restricted to end-zone areas in the receptive field and to the alignment of test line and surrounding lines (Knierim \& Van Essen, 1992; Van Essen et al., 1989). Thus, although the assumed mechanisms of interrupted line continuation and local orientation contrast are functionally similar and indistinguishable to some extent, orientation contrast seems to provide a more plausible explanation of the perceptual phenomena seen.

\section{Figural Synthesis}

The detection (and localization) of local orientation contrast is a necessary requirement for the grouping and figural synthesis of texture elements. Orientation itself was not seen to play an important role, as far as the analysis for similarity is concerned. However, the additional effects from alignment in texture segmentation (Experiment 2) and in grouping (Experiment 6) show that the detection of configurations is not completely independent of the orientation of line elements but is notably influenced by the alignment of local line orientation with figure borders (provided that the line elements are sufficiently distinct from their neighbors). Variations in line orientation may cause different percepts of the same figure, as is demonstrated in Figure 13. Whereas in Figure 13A, the salient elements seem to form a circle, they obviously form a square in Figure 13B, although the elements have not changed their positions but have only switched their orientation. Important cues for identifying a figure are the corners of that figure. This suggests that elements whose orientations coincide with the outlines of a figure near its corners would provide the most vivid impression of that figure, as can indeed be seen in Figure 13C. Interestingly, according to this study, corners are always more salient than continuous lines, because of the direct orientation contrast of their flanks. This may explain why corners are so important in vision: they pop out. In addition, they provide the necessary information for evaluating the outlines of a figure and tell the visual system in which direction it might be worthwhile to search for further salient elements.

\section{Other Features}

There are many more visual cues that induce segmentation and pop-out than just differences in orientation. As mentioned above, I have been testing some of them recently, using paradigms similar to those in this study. Interestingly, although many of the statements made here
A

$\left[\begin{array}{llllllllllllllll}\hline & 1 & 1 & 1 & 1 & 1 & 1 & 1 & 1 & 1 & 1 & 1 & 1 & 1 & 1 & 1 \\ 1 & 1 & 1 & 1 & 1 & 1 & 1 & 1 & 1 & 1 & 1 & 1 & 1 & 1 & 1 \\ 1 & 1 & 1 & 1 & 1 & 1 & 1 & 1 & 1 & 1 & 1 & 1 & 1 & 1 & 1 & 1 \\ 1 & 1 & 1 & 1 & 1 & 1 & 1 & 1 & 1 & 1 & 1 & 1 & 1 & 1 & 1 & 1 \\ 1 & 1 & 1 & 1 & 1 & 1 & 1 & 1 & 1 & 1 & 1 & 1 & 1 & 1 & 1 & 1 \\ 1 & 1 & 1 & 1 & 1 & 1 & 1 & 1 & 1 & 1 & 1 & 1 & 1 & 1 & 1 & 1 \\ 1 & 1 & 1 & 1 & 1 & 1 & 1 & 1 & 1 & 1 & 1 & 1 & 1 & 1 & 1 & 1 \\ 1 & 1 & 1 & 1 & 1 & 1 & 1 & 1 & 1 & 1 & 1 & 1 & 1 & 1 & 1 & 1 \\ 1 & 1 & 1 & 1 & 1 & 1 & 1 & 1 & 1 & 1 & 1 & 1 & 1 & 1 & 1 & 1 \\ 1 & 1 & 1 & 1 & 1 & 1 & 1 & 1 & 1 & 1 & 1 & 1 & 1 & 1 & 1 & 1 \\ 1 & 1 & 1 & 1 & 1 & 1 & 1 & 1 & 1 & 1 & 1 & 1 & 1 & 1 & 1 & 1 \\ 1 & 1 & 1 & 1 & 1 & 1 & 1 & 1 & 1 & 1 & 1 & 1 & 1 & 1 & 1 & 1 \\ 1 & 1 & 1 & 1 & 1 & 1 & 1 & 1 & 1 & 1 & 1 & 1 & 1 & 1 & 1 & 1 \\ 1 & 1 & 1 & 1 & 1 & 1 & 1 & 1 & 1 & 1 & 1 & 1 & 1 & 1 & 1 & 1 \\ 1 & 1 & 1 & 1 & 1 & 1 & 1 & 1 & 1 & 1 & 1 & 1 & 1 & 1 & 1 & 1 \\ 1 & 1 & 1 & 1 & 1 & 1 & 1 & 1 & 1 & 1 & 1 & 1 & 1 & 1 & 1 & 1\end{array}\right]$

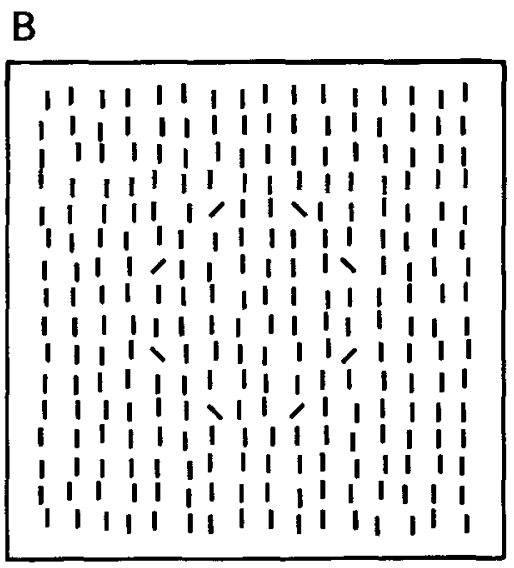

C

Figure 13. Influence of line orientation on the percept of configurational grouping. In all patterns, eight elements pop out because of local orientation contrast. They seem to resemble a circle in panel $A$ and a square in panel $B$, although the elements' positions are identical and only line orientations are exchanged. The impression of a square is even stronger in panel $\mathrm{C}$, where line elements mark the corners of such a figure. Thus, local alignment of line elements with the outline of the global figure has a considerable impact on the percept in these patterns. 
can be applied to the processing of other stimulus properties, some cannot. Motion, for example, was found to have properties very similar to those of line orientation, with segmentation and grouping being based primarily on local differences in the pattern (Nothdurft, 1991b). Color, however, as well as luminance and depth, seems to behave differently in some respects.

\section{CONCLUSIONS}

In this paper, I have shown that texture segmentation from orientation is not based on element similarity within a texture area but is based on local dissimilarities at texture borders.

I have also shown that the percept of an orientation target popping out from its context ("the odd man out" paradigm) can be related to target saliency, which was found to depend on local orientation contrast from the overall variation of line orientation in the pattern, and not orientation per se. Searching for lines at a certain orientation could only be done fast ("in parallel") if elements were sufficiently distinct from their neighbors. Local dissimilarity, therefore, is a prerequisite for pop-out and fast visual search.

Even the grouping of orientation targets was shown to depend primarily on the saliency rather than on the similarity of texture elements. Nonsalient elements did not group. And, in preattentive vision, salient similar line elements did not group any better than salient dissimilar ones. Apparently it is only at a second (probably attentive) stage of analysis that observers can see groups of similar lines and voluntarily shift between such groups.

\section{REFERENCES}

Bacon, W. F., \& EGeTh, H. E. (1991). Local processes in preattentive feature detection. Journal of Experimental Psychology: Human Perception \& Performance, 17, 77-90.

BECK, J. (1966a). Effect of orientation and of shape similarity on perceptual grouping. Perception \& Psychophysics, 1, 300-302.

BECK, J. (1966b). Perceptual grouping produced by changes in orientation and shape. Science, 154, 538-540.

BECK, J. (1967). Perceptual grouping produced by line figures. Perception \& Psychophysics, 2, 491-495.

BEck, J. (1972). Similarity grouping and peripheral discriminability under uncertainty. American Journal of Psychology, 85, 1-19.

BECK, J. (1982). Textural segmentation. In J. Beck (Ed.), Organization and representation in perception (pp. 285-317). Hillsdale, NJ: Erlbaum.

Beck, J., Prazdny, K., Rosenfeld, A. (1983). A theory of textural segmentation. In J. Beck, B. Hope, \& A. Rosenfeld (Eds.), Human and machine vision (pp. 1-38). London: Academic Press.

Beck, J., Rosenfeld, A., \& IVRY, R. (1989). Line segregation. Spatial Vision, 4, 75-101.

BERGEN, J. R., ADELSON, E. H. (1988). Early vision and texture perception. Nature, 333, 363-364.

DunCan, J., Humphreys, G. W. (1989). Visual search and stimulus similarity. Psychological Review, 96, 433-458.

FOSTER, D. H., \& WARD, P. A. (1991a). Asymmetries in oriented-line detection indicate two orthogonal filters in early vision. Proceedings of the Royal Society of London: Series B, 243, 75-81.
Foster, D. H., W WRD, P. A. (1991b). Horizontal-vertical filters in early vision predict anomalous line-orientation frequencies. Proceedings of the Royal Society of London: Series B, 243, 83-86.

GILBERT, C. D. (1985). Horizontal integration in the neocortex. Trends in Neuroscience, 8, 160-165.

GILBERT, C. D., WIESEL, T. N. (1981). Laminar specialization and intracortical connections in cat primary visual cortex. In F. O. Schmitt, F. G. Warden, G. Adelman, \& S. G. Dennis (Eds.), The organization of the cerebral cortex (pp. 163-194), Cambridge, MA: MIT Press.

Gilbert, C. D., Wiesel, T. N. (1989). Columnar specificity of intrinsic horizontal and corticocortical connections in cat visual cortex. Journal of Neuroscience, 9, 2432-2442.

Gurnsey, R., \& Browse, R. A. (1987). Micropattern properties and presentation conditions influencing visual texture discrimination. Perception \& Psychophysics, 41, 239-252.

Hallett, P. E., HofmanN, M. I. (1991). Segregation of some meshderived textures evaluated by free viewing. Vision Research, 31, 1701-1716.

JuLESZ, B. (1975). Experiments in the visual perception of texture. Scientific American, 232(4), 34-43.

Julesz, B. (1984). A brief outline of the texton theory of human vision. Trends in Neuroscience, 7, 41-45.

JulEsz, B. (1986). Texton gradients: The texton theory revisited. Biological Cybernetics, 54, 245-251.

JULESz, B., \& BERGEN, J. R. (1983). Textons, the fundamental elements in preattentive vision and perception of textures. Bell System Technical Journal, 62, 1619-1645.

Knierim, J. J., \& VAN Essen, D. C. (1992). Neuronal responses to static texture patterns in area V1 of the alert macaque monkey. Journal of Neurophysiology, 67, 961-980.

LINK, N., ZuCKER, S. W. (1987). Sensitivity to corners in flow patterns. Spatial Vision, 2, 233-244.

Mitchinson, G., \& Crick, F. (1982). Long axons within the striate cortex: Their distribution, orientation and patterns of connection. Proceedings of the National Academy of Sciences of the United States of America, 79, 3661-3665.

MoraGlia, G. (1989). Display organization and the detection of horizontal line segments. Perception \& Psychophysics, 45, 265-272.

NothDURFT, H. C. (1985a). Orientation sensitivity and texture segmentation in patterns with different line orientation. Vision Research, 25, $551-560$.

NothduRFt, H. C. (1985b). Sensitivity for structure gradient in texture discrimination tasks. Vision Research, 25, 1957-1968.

NothDURFT, H. C. (1990). Texton segregation by associated differences in global and local luminance distribution. Proceedings of the Royal Society of London: Series B, 239, 295-320.

NothduRFT, H. C. (1991a). Different effects from spatial frequency masking in texture segregation and texton detection tasks. Vision $\mathbf{R e}$ search, 31, 299-320.

NothduRF, H. C. (1991b). The role of local contrast in pop-out of orientation, motion and color. Investigative Opththalmology \& Visual Science, 32, 714.

NothduRFT, H. C. (1991c). Texture segmentation and pop-out from orientation contrast. Vision Research, 31, 1073-1078.

Northdurft, H. C. (in press). Fast detection of targets. Perception.

Olson, R. K., ATTNEAVE, F. (1970). What variables produce similarity grouping? American Joumal of Psychology, 83, 1-21

OR, Y. H., ZuCKER, S. W. (1989). Texture fields and texture flows: Sensitivity to differences. Spatial Vision, 4, 131-139.

Parlitz, D., Nothdurf, H. C. (1990). Does pop-out of orientation or motion produce express saccades? In N. Elsner \& G. Roth (Eds.), Brain, perception, cognition (p. 258). Stuttgart: Thieme Verlag.

SAGI, D, \& Julesz, B. (1985). "Where" and "what" in vision. Science, 228, 1217-1219.

SAGI, D., JuLESZ, B. (1987). Short-range limitations on detection of feature differences. Spatial Vision, 2, 39-49.

Treisman, A. (1985). Preattentive processing in vision. Computer Vision, Graphics, \& Image Processing, 31, 156-177. 
Treisman, A. (1986). Features and objects in visual processing. Scientific American, 255(11), 106-115.

Treisman, A. (1988). Features and objects: The fourteenth Bartlett Memorial Lecture. Quarterly Journal of Experimental Psychology, 40A, 201-237.

Treisman, A., \&ormican, S. (1988). Feature analysis in early vision: Evidence from search asymmetries. Psychological Review, 95 , 15-48.

Van Essen, D. C., DeYoe, E. A., Olavarria, J., Knierim, J., Fox, J., SAGI, D., JULESZ, B. (1989). Neural responses to static and moving texture patterns in visual cortex of the Macaque monkey. In D. M. K. Lam \& C. D. Gilbert (Eds.), Neural mechanisms of visual perception (pp. 137-154). Woodlands, TX: Portfolio Publishing.
Von der Heydt, R., Peterhans, E., Baumgartner, G. (1984). Illusory contours and cortical neuron responses. Science, 224. $1260-1262$.

WERTHEIMER, M. (1923). Untersuchungen zur Lehre von der Gestalt: II. Psychologische Forschung, 4, 301-350.

Wolfe, J. M., Friedman-Hill, S. R., Stewart, M. I., O'Connell, K. M. (1992). The role of categorization in visual search for orientation. Joumal of Experimental Psychology: Human Perception \& Per formance, 18, 34-49.

(Manuscript received November 1, 1991 ; revision accepted for publication April 11, 1992.) 